\title{
Wave-front tracking for the equations of non-isentropic gas dynamics
}

\author{
Fumioki Asakura • Andrea Corli
}

Received: 6 May 2013 / Accepted: 19 November 2013 / Published online: 6 December 2013

(C) Fondazione Annali di Matematica Pura ed Applicata and Springer-Verlag Berlin Heidelberg 2013

\begin{abstract}
We study the model equations of polytropic gas dynamics, which constitute a system of three hyperbolic conservation laws. Global in time BV solutions were obtained by Liu (Indiana Univ Math J 26(1):147-177, 1977) provided that $(\gamma-1)$ times the total variation of the initial data is sufficiently small; here $\gamma$ is the adiabatic coefficient. The aim of this paper is to give an alternative proof by exploiting the Dafermos-Bressan-Risebro wave-front tracking scheme. An original feature is the use of the path decomposition method to obtain pathwise estimates of the approximate solutions; these estimates show the decay properties of the solutions and play a crucial role in proving the stability of the wave-front tracking scheme.
\end{abstract}

Keywords Hyperbolic systems of conservation laws - Gas dynamics equations · Wave-front tracking

Mathematics Subject Classification $35 \mathrm{~L} 65 \cdot 35 \mathrm{~L} 67 \cdot 76 \mathrm{~N} 15$

\section{Introduction}

The equations of gas dynamics in one-space dimension are given in Lagrangian coordinates by

$$
\left\{\begin{array}{l}
v_{t}-u_{x}=0 \\
u_{t}+p_{x}=0 \\
\left(\mathcal{E}+\frac{1}{2} u^{2}\right)_{t}+(p u)_{x}=0
\end{array}\right.
$$

F. Asakura

Department of Engineering Science, Osaka Electro-communication University, Neyagawa, Osaka, Japan e-mail: asakura@isc.osakac.ac.jp

A. Corli (凶)

Department of Mathematics and Computer Science, University of Ferrara, Ferrara, Italy

e-mail: andrea.corli@unife.it 
for $(x, t) \in \mathbb{R} \times \mathbb{R}_{+}$. Here above, $u$ is the velocity, $p$ the pressure, $v$ the specific volume, and $\mathcal{E}$ the internal energy; $p$ and $v$ are positive quantities. Temperature and entropy are denoted by $\Theta, S$, respectively, and satisfy the first and second law of thermodynamics: $d \mathcal{E}=\Theta d S-p d v$, [10]. The gas is assumed to be ideal, i.e., $p v=R \Theta$, and polytropic: $\mathcal{E}=C_{v} \Theta+\mathcal{E}_{0}$; here, $R, C_{v}$ and $\mathcal{E}_{0}$ are constants. As a consequence, the entropy $S$ is expressed as

$$
S=C_{v}(\log p+\gamma \log v)+\text { const, }
$$

where $\gamma=1+R / C_{v}>1$. By setting $\mathcal{E}_{0}=-\frac{a^{2}}{\gamma-1}$, so that $\mathcal{E}$ makes sense for $\gamma \rightarrow 1$, we have

$$
\mathcal{E}=\frac{p v-a^{2}}{\gamma-1}, \quad p=a^{2} v^{-\gamma} e^{\frac{\gamma-1}{R} S} .
$$

In the limit case $\gamma=1$, one has $p=a^{2} v^{-1}$ and $\mathcal{E}=a^{2}(-\log v+S / R)$; hence, the system (1.1) coincides with the equation of isothermal gas dynamics.

We shall discuss the existence of solutions, defined globally in time, to the initial-value problem for the equations (1.1). The initial data are given by

$$
\left.(v, u, S)\right|_{t=0}=(\bar{v}(x), \bar{u}(x), \bar{S}(x)),
$$

with

$$
\bar{v}(x) \geq v_{*}>0
$$

and $\bar{v}, \bar{u}, \bar{S} \in \mathrm{BV}(\mathbb{R})$, the space of functions having bounded total variation. We denote by $\mathrm{TV}(f)$ the total variation of a function $f \in \mathrm{BV}(\mathbb{R})$.

If the total variation of the initial data is sufficiently small, then Glimm's existence theorem [11] applies for any $\gamma \geq 1$ and guarantees the existence of global solutions. Hence, the issue is to study the case of initial data whose total variation is not necessarily small. The following results are by now classic.

Theorem 1.1 (Liu [15]) Assume $1<\gamma \leq \frac{5}{3}$ and (1.3). If $(\gamma-1) \mathrm{TV}(\bar{v}, \bar{u}, \bar{S})$ is sufficiently small, then there exists a global in time BV-solution to (1.1)-(1.2).

Theorem 1.2 (Nishida [17]) Assume $\gamma=1$ and (1.3). If the total variation $\mathrm{TV}(\bar{v}, \bar{u}, \bar{S})$ of the initial data is finite, then there exists a global in time BV-solution to (1.1)-(1.2).

Of course, if $\gamma=1$, then the two first equations in (1.1) decouple from the third one and the entropy $S$ is computed by means of $v$ and the initial data $\bar{S}$. Another celebrated paper, due to Nishida and Smoller [18], dealt previously with the system of isentropic gas dynamics and a result analogous to Theorem 1.1 was proved. Later on, Temple [22] provided a different proof of Theorem 1.1, based on the observation that the polytropic gas model $1<\gamma \leq \frac{5}{3}$ can be treated as a perturbation of the isothermal model $\gamma=1$. All of these results were proved by exploiting the random choice method due to Glimm [11].

In recent years, the wave-front tracking scheme, which was initiated by Dafermos [8] for a single conservation laws and then developed by Bressan [6,7], and Risebro [19] for hyperbolic systems of conservation laws, has been proved a better alternative to Glimm's scheme. This approach has the following advantages:

- Free from random sampling;

- Based on simple interaction estimates;

- Useful in studying asymptotic properties;

- Appropriate for establishing the continuous dependence on the initial data. 
The wave-front tracking scheme was successfully applied to the case of isentropic gas dynamics by Asakura [3]. About continuous dependence both in the isentropic case and for the full system (1.1), we also refer to [13].

This paper is the natural continuation of the analysis begun in Asakura [3]. Our main goal is to provide a proof to both theorems above by the front-tracking scheme. In doing this, a difficult issue is the control of the total variation of the approximate solutions; to this end, we exploit a rather new technique, the path decomposition method, which was first fully exploited by Asakura in [3]. Notice that, although the notion of path was introduced by Temple and Young [21], the idea of the decomposition indeed goes back to Asakura [2]. It consists, roughly speaking, in the following.

Paths are broken lines, in the $x t$-plane, which follow shock and entropy fronts. Each front is decomposed into finitely many segments, whose positions coincide with that of the front but belong to different paths. A notion of strength is then introduced for each segment, in such a way that the strength of a wave is the sum of the strengths of the segments that decompose it. A generation order is also defined for each segment of the path. The goal of this apparently complicated construction is to deal separately with primary paths and secondary paths. In the former case, the strength is decreasing along the path and then the control of the variation is trivial. The latter case takes into account, for instance, both the "new" shocks generated through an interaction (for example, shocks that belong to families different from the interacting ones) and a part of those shocks whose strength is amplified in the interaction (this is the case, for instance, of a shock emerging from the interaction of two shocks, all of them belonging to the same family).

Nishida's lemma [16] states that, in the case of isothermal gas dynamics, the total amount of shock waves in Glimm approximate solutions does not increase. We shall obtain the estimates of the approximate solutions along paths, showing that the strength of each path decreases at the rate of $c^{n-1}$ (for $0<c<1$ ) as $n$, the generation order, increases. This is the "pathwise" version for system (1.1) of Nishida's lemma and plays a crucial role in proving both the stability of the wave-front tracking scheme and the decay property of the weak entropy solutions (see Glimm and Lax [12]). If $1<\gamma \leq \frac{5}{3}$, we also introduce secondary waves and show that the total amount of these waves is $(\gamma-1)$ times the interaction potential; this leads to a further understanding both of the assumption made in Theorem 1.1 and of the aforementioned perturbation method of Temple [22].

The main result of this paper is the following.

Theorem 1.3 Under the same assumptions of Theorem 1.1, the wave-front tracking scheme is stable and provides a global BV-solution to (1.1)-(1.2).

As we emphasized above, the interest of this result lies more in the techniques of proof than in its bare statement.

The system (1.1) is close to the following one, which arises in the modelling of phase transitions in fluids:

$$
\left\{\begin{array}{l}
v_{t}-u_{x}=0, \\
u_{t}+p(v, \lambda)_{x}=0, \\
\lambda_{t}=0
\end{array}\right.
$$

Global BV-solutions are obtained by Amadori and Corli [1], Asakura and Corli [5], Holden, Risebro and Sande [14] for $p=a^{2}(\lambda) v^{-1}$ in [1,5] and $p=a^{2} v^{-\lambda}$ in [14]. System (1.4) is simpler than (1.1) in the sense that if the initial datum $\bar{\lambda}$ is constant in an interval $\left[x_{0}, x_{1}\right]$, then in that region it reduces to the system of isothermal or isentropic gas dynamics. However, the study of (1.4) is somewhat more difficult than that of (1.1), because the former lacks of any 
appropriate parameter as $\epsilon=\frac{1}{2}(\gamma-1)$ in the latter, where $\epsilon$ times the total variation of the initial data is assumed arbitrarily small. However, some basic ideas and methods developed in [5] are adopted in the present paper. We emphasize that in [5], only shock fronts (and not entropy fronts, as it is also the case here) gave rise to paths.

The paper is organized as follows. In Sect. 2, we quickly introduce the notation concerning the wave curves and the Riemann problem $[7,9,20]$. Then, the front-tracking scheme is described in Sect. 3 following Bressan [7]. Section 4 deals with the (very) technical subject of the local interaction estimates. The main result of this section, namely Lemma 4.3, was first given by Liu [15]; however, the interested reader can find fully detailed proofs in [4]. In Sect. 5, we finally enter the core of the paper by proving the global interaction estimates, which parallel those of Liu [15] but are suitably adapted to our different scheme. We define paths and their strengths in Sect. 6; a precise description of the decomposition by paths is given there. Estimates along paths are obtained in Sect. 7 and the stability of the front-tracking scheme is finally proved in Sect. 8. Our estimates also imply the asymptotic stability of the BV-solutions obtained in this paper (see Asakura [3, Section 8]).

\section{The Riemann problem}

The quantity $\eta=S / R$ is called the dimensionless entropy. As we shall see in (2.8), system (1.1) admits stationary waves connecting states with $p_{+}=p_{-}, u_{+}=u_{-}, \eta_{+} \neq \eta_{-}$; then, it is useful to choose $U=(p, u, \eta)$ as independent variables. Note that $v$ can be written by using $p$ and $\eta$; we have

$$
v=a^{\frac{2}{\gamma}} e^{\frac{\gamma-1}{\gamma} \eta} p^{-\frac{1}{\gamma}} \text { and } \sqrt{-v_{p}(p, \eta)}=\gamma^{-\frac{1}{2}} a^{\frac{1}{\gamma}} e^{\frac{\gamma-1}{2 \gamma} \eta} p^{-\frac{\gamma+1}{2 \gamma}} .
$$

The quasi-linear equations associated with (1.1) are

$$
p_{t}-\frac{u_{x}}{v_{p}}=0, \quad u_{t}+p_{x}=0, \quad \eta_{t}=0 .
$$

From these equations, we find that the characteristic speeds are

$$
\lambda_{1}(U)=-\frac{1}{\sqrt{-v_{p}(p, \eta)}}, \quad \lambda_{2}(U)=\frac{1}{\sqrt{-v_{p}(p, \eta)}}, \quad \lambda_{0}(U)=0
$$

and the corresponding characteristic vector fields can be written as

$$
\begin{gathered}
R_{1}(U)={ }^{t}\left(1,-\sqrt{-v_{p}(p, \eta)}, 0\right), \quad R_{2}(U)={ }^{t}\left(1, \sqrt{-v_{p}(p, \eta)}, 0\right), \\
R_{0}(U)={ }^{t}(0,0,1) .
\end{gathered}
$$

Setting $\epsilon=\frac{\gamma-1}{2}$, we can write the forward 1-rarefaction curve $\widehat{\mathcal{R}}_{1}^{F}\left(U_{0}\right)$ and the backward 2-rarefaction curve $\widehat{\mathcal{R}}_{2}^{B}\left(U_{0}\right)$ issuing from $U_{0}$ as

$$
\begin{aligned}
& \widehat{\mathcal{R}}_{1}^{F}\left(U_{0}\right):\left\{\begin{array}{l}
u-u_{0}=-\frac{\sqrt{\gamma} a^{\frac{1}{\gamma}}}{\epsilon} e^{\frac{\epsilon}{\gamma} \eta_{0}}\left(p^{\frac{\epsilon}{\gamma}}-p_{0}^{\frac{\epsilon}{\gamma}}\right), \quad p \leq p_{0}, \\
\eta-\eta_{0}=0,
\end{array}\right. \\
& \widehat{\mathcal{R}}_{2}^{B}\left(U_{0}\right):\left\{\begin{array}{l}
u-u_{0}=\frac{\sqrt{\gamma} a^{\frac{1}{\gamma}}}{\epsilon} e^{\frac{\epsilon}{\gamma} \eta_{0}}\left(p^{\frac{\epsilon}{\gamma}}-p_{0}^{\frac{\epsilon}{\gamma}}\right), \quad p \leq p_{0} . \\
\eta-\eta_{0}=0 .
\end{array}\right.
\end{aligned}
$$

These curves are integral curves of $R_{j}(U)(j=1,2)$, respectively. If $U \in \widehat{\mathcal{R}}_{1}^{F}\left(U_{0}\right)$, there is a 1-rarefaction wave connecting $U_{0}$ and $U$; if $U \in \widehat{\mathcal{R}}_{2}^{B}\left(U_{0}\right)$, there is a 2-rarefaction wave connecting $U$ and $U_{0}$. 
A self-similar jump discontinuity having the form

$$
U(x, t)= \begin{cases}U_{-} & \text {for } x<s t \\ U_{+} & \text {for } x>s t\end{cases}
$$

is a weak solution of (1.1) if and only if the constant states $U_{-}$and $U_{+}$satisfy the RankineHugoniot condition

$$
\left\{\begin{array}{l}
\mathcal{E}_{+}-\mathcal{E}_{-}+\frac{1}{2}\left(p_{+}+p_{-}\right)\left(v_{+}-v_{-}\right)=0 \\
\left(u_{+}-u_{-}\right)^{2}=-\left(p_{+}-p_{-}\right)\left(v_{+}-v_{-}\right)
\end{array}\right.
$$

where the shock speed $s$ satisfies $s^{2}=-\frac{p_{+}-p_{-}}{v_{+}-v_{-}}$. For a polytropic gas, the Rankine-Hugoniot condition is equivalent to

$$
\left\{\begin{aligned}
e^{(\gamma-1)\left(\eta_{+}-\eta_{-}\right)} & =\left(\frac{p_{+}}{p_{-}}\right)\left\{\frac{(\gamma-1) p_{+}+(\gamma+1) p_{-}}{(\gamma+1) p_{+}+(\gamma-1) p_{-}}\right\}^{\gamma}, \\
\left(u_{+}-u_{-}\right)^{2} & =\frac{2 v_{-}\left(p_{+}-p_{-}\right)^{2}}{(\gamma+1) p_{+}+(\gamma-1) p_{-}} .
\end{aligned}\right.
$$

If $p_{+} \neq p_{-}$, we have two branches of solutions to (2.3):

$$
u_{+}-u_{-}= \pm \sqrt{\frac{2 v_{-}}{(\gamma+1) p_{+}+(\gamma-1) p_{-}}}\left(p_{+}-p_{-}\right), \quad s= \pm \sqrt{-\frac{p_{+}-p_{-}}{v_{+}-v_{-}}} .
$$

Let us fix $p_{-}$and $\eta_{-}$. When considering $\eta_{+}$as a function of $p_{+}$, we have

$$
\frac{d \eta_{+}}{d p_{+}}=\frac{(\gamma+1)\left(p_{+}-p_{-}\right)^{2}}{p_{+}\left\{(\gamma-1) p_{+}+(\gamma+1) p_{-}\right\}\left\{(\gamma+1) p_{+}+(\gamma-1) p_{-}\right\}}>0,
$$

which shows that $\eta_{+}>\eta_{-}$if and only if $p_{+}>p_{-}$. Since the entropy must increase as time goes on, the physically relevant branches are

$$
p_{+}>p_{-} \text {for } s<0 \text { and } p_{+}<p_{-} \text {for } s>0 .
$$

A jump discontinuity (2.2) lying on a Hugoniot branch satisfying (2.6) is called a shock wave, and the line of discontinuity $x=s t$ is referred to as a shock front. More precisely, by setting

$$
\begin{aligned}
\mathcal{G}\left(p_{0}, p ; \eta_{0}\right) & =\frac{a^{\frac{1}{\gamma}} e^{\frac{\epsilon}{\gamma} \eta_{0}}\left(p-p_{0}\right)}{p_{0}^{\frac{1}{2 \gamma}}\left\{(1+\epsilon) p+\epsilon p_{0}\right\}^{\frac{1}{2}}}, \\
\mathcal{H}\left(p_{0}, p\right) & =\frac{1}{2 \epsilon} \log \left[\left(\frac{p}{p_{0}}\right)\left\{\frac{\epsilon p+(1+\epsilon) p_{0}}{(1+\epsilon) p+\epsilon p_{0}}\right\}^{\gamma}\right],
\end{aligned}
$$

we define the forward 1-shock curve $\widehat{\mathcal{S}}_{1}^{F}\left(U_{0}\right)$ and the backward 2-shock curve $\widehat{\mathcal{S}}_{2}^{B}\left(U_{0}\right)$ issuing from $U_{0}$ as

$$
\begin{aligned}
& \widehat{\mathcal{S}}_{1}^{F}\left(U_{0}\right):\left\{\begin{array}{l}
u-u_{0}=-\mathcal{G}\left(p_{0}, p ; \eta_{0}\right), \\
\eta-\eta_{0}=\mathcal{H}\left(p_{0}, p\right),
\end{array} \quad p>p_{0},\right. \\
& \widehat{\mathcal{S}}_{2}^{B}\left(U_{0}\right):\left\{\begin{array}{l}
u-u_{0}=\mathcal{G}\left(p_{0}, p ; \eta_{0}\right), \quad p>p_{0} . \\
\eta-\eta_{0}=\mathcal{H}\left(p_{0}, p\right),
\end{array}\right.
\end{aligned}
$$

At last, if $p_{+}=p_{-}$, we have an entropy wave

$$
u_{+}=u_{-}, \quad p_{+}=p_{-}, \quad \eta_{+} \neq \eta_{-}, \quad s=0,
$$


which coincides with the integral curve $R_{0}\left(U_{-}\right)$when $\eta_{+}$varies. This type of discontinuity is also called a contact discontinuity.

We define the forward 1-wave curve $\widehat{\mathcal{W}}_{1}^{F}\left(U_{L}\right)=\widehat{\mathcal{R}}_{1}^{F}\left(U_{L}\right) \cup \widehat{\mathcal{S}}_{1}^{F}\left(U_{L}\right)$ and the backward 2wave curve $\widehat{\mathcal{W}}_{2}^{B}\left(U_{R}\right)=\widehat{\mathcal{R}}_{2}^{B}\left(U_{R}\right) \cup \widehat{\mathcal{S}}_{2}^{B}\left(U_{R}\right)$; the 0 -wave curves are just the integral curves $R_{0}$. Each wave curve constitutes a $C^{2}$-curve with Lipschitz-continuous second derivative; they represent all realizable rarefaction, shock, and entropy waves. More precisely, if $(p, u, \eta) \in$ $\widehat{\mathcal{W}}_{1}^{F}\left(U_{L}\right)$, then there is a 1-rarefaction or shock wave connecting $\left(p_{L}, u_{L}, \eta_{L}\right)$ and $(p, u, \eta)$; if $(p, u, \eta) \in \widehat{\mathcal{W}}_{2}^{B}\left(U_{R}\right)$, there is a 2-rarefaction or shock wave connecting $(p, u, \eta)$ and $\left(p_{R}, u_{R}, \eta_{R}\right)$. The projections of the curves $\widehat{\mathcal{W}}_{1}^{F}\left(U_{L}\right)$ and $\widehat{\mathcal{W}}_{2}^{B}\left(U_{R}\right)$ on the $p u$-plane are denoted by $\mathcal{W}_{1}^{F}\left(U_{L}\right)$ and $\mathcal{W}_{2}^{B}\left(U_{R}\right)$, respectively.

The Riemann problem, i.e., the initial-value problem for (1.1) with piecewise constant initial data

$$
U(x, 0)= \begin{cases}U_{L} & \text { if } x<0 \\ U_{R} & \text { if } x>0\end{cases}
$$

is solved in the following way. Let $\left(p_{L}, u_{L}, \eta_{L}\right)$ and $\left(p_{R}, u_{R}, \eta_{R}\right)$ be given Riemann data. If the curves $\mathcal{W}_{1}^{F}\left(U_{L}\right)$ and $\mathcal{W}_{2}^{B}\left(U_{R}\right)$, have an intersection point $\left(p_{m}, u_{m}\right)$, then the state $\left(p_{m}, u_{m}, \eta_{m}^{-}\right) \in \widehat{\mathcal{W}}_{1}^{F}\left(U_{L}\right)$ and $\left(p_{m}, u_{m}, \eta_{m}^{+}\right) \in \widehat{\mathcal{W}}_{2}^{B}\left(U_{R}\right)$ are connected by an entropy wave. Since the sound speed is expressed as

$$
c=\sqrt{\gamma p v}=\sqrt{\gamma} a^{\frac{1}{\gamma}} e^{\frac{\epsilon}{\gamma} \eta} p^{\frac{\epsilon}{\gamma}},
$$

we have the following well-known theorem, where uniqueness is understood in the sense of Smoller [20, Theorem 18.6].

Theorem 2.1 The Riemann problem for (1.1) with data (2.9) has a unique solution if

$$
u_{R}-u_{L}<\frac{c_{L}+c_{R}}{\epsilon} \text {. }
$$

\section{The wave-front tracking scheme}

The wave-front tracking scheme provides a way for constructing approximate solutions to the system (1.1) with initial data

$$
U(x, 0)=\bar{U}(x)
$$

in the class $\mathrm{BV}(\mathbb{R})$. We quickly sketch here the main steps, referring the reader to [7] for details. We define

$$
\eta_{*}=\inf \bar{\eta}(x) .
$$

Let $h$ be a positive number. We approximate the initial data $\bar{U}(x)$ by a step function $\bar{U}^{h}(x)$ having finitely many jumps; moreover, we may assume $\eta_{*} \leq \min \bar{\eta}^{h}(x)$.

Let $x_{1}<\cdots<x_{M}$ be the points of discontinuity of $\bar{U}^{h}(x)$. At each $x_{m}$, we solve the Riemann problem with initial data $U_{L}=\bar{U}^{h}\left(x_{m}-0\right), U_{R}=\bar{U}^{h}\left(x_{m}+0\right)$ in an approximate way: every rarefaction wave is substituted by several small fans consisting of constant states and jump discontinuities separating them. This is called the accurate Riemann solver.

The issue is how to extend the approximate solution $U^{h}$ after a wave interaction. To avoid the breakdown due to the possible divergence of the jump discontinuities within a finite time, a simplified Riemann solver must be introduced. It consists in prolonging each interacting 
wave, say $\theta^{\prime}$ and $\theta^{\prime \prime}$, with a wave of the same family and size; if the fronts belong to the same family, they are prolonged as a single front of size $\theta^{\prime}+\theta^{\prime \prime}$. Since the waves in general do not commute, a non-physical front is introduced with a sufficiently high-speed $\hat{\lambda}$.

Now, we describe how the two solvers are used in the construction of $U^{h}$. Recall that the amount of waves emerging from an interaction is estimated by the product $\left|\theta^{\prime} \theta^{\prime \prime}\right|$ of the strengths of the incoming waves. Then, we fix a threshold $\rho>0$ and extend $U^{h}$ past an interaction of two waves $\theta^{\prime}$ and $\theta^{\prime \prime}$ at $(x, t)$ as follows: if $\left|\theta^{\prime} \theta^{\prime \prime}\right| \geq \rho$, then we use the accurate Riemann solver with data $U^{h}(x, t-0)$, else if $\left|\theta^{\prime} \theta^{\prime \prime}\right|<\rho$, we use the simplified Riemann solver, with the proviso that if one of the waves is an entropy wave, then we use the former solver if $M_{0}\left|\theta^{\prime} \theta^{\prime \prime}\right| \geq \rho$ and the latter if $M_{0}\left|\theta^{\prime} \theta^{\prime \prime}\right| \leq \rho$. Here, $M_{0}$ is the constant defined in (5.28).

The above procedure gives an approximate solution up to some time $T>0$; in order to prove that $U^{h}$ is defined for $t \in(0,+\infty)$, we shall show that the number of interactions remains finite for any time.

\section{Interaction of two incoming waves}

We define $\bar{p}_{*}=\inf \bar{p}(x)$. We shall show in Lemma 5.2 that there exists $p_{*}, p^{*}$ and $H$ such that all waves under consideration are in the region

$$
0<p_{*} \leq p \leq p^{*}, \quad 0 \leq \eta-\eta_{*} \leq H,
$$

if $\epsilon \operatorname{TV}(\bar{p}, \bar{u}, \bar{\eta})$ is sufficiently small. Therefore, in the following, we assume that (4.1) holds.

We introduce the Riemann invariants with respect to $\eta_{*}$

$$
w=u-\frac{\sqrt{\gamma} a^{\frac{1}{\gamma}}}{\epsilon} e^{\frac{\epsilon}{\gamma} \eta_{*}}\left(p^{\frac{\epsilon}{\gamma}}-\bar{p}_{*}^{\frac{\epsilon}{\gamma}}\right), \quad z=u+\frac{\sqrt{\gamma} a^{\frac{1}{\gamma}}}{\epsilon} e^{\frac{\epsilon}{\gamma} \eta_{*}}\left(p^{\frac{\epsilon}{\gamma}}-\bar{p}_{*}^{\frac{\epsilon}{\gamma}}\right)
$$

and set

$$
\tau=\frac{z-w}{2}=\frac{\sqrt{\gamma} a^{\frac{1}{\gamma}}}{\epsilon} e^{\frac{\epsilon}{\gamma} \eta_{*}}\left(p^{\frac{\epsilon}{\gamma}}-\bar{p}_{*}^{\frac{\epsilon}{\gamma}}\right) .
$$

Remark 4.1 In Liu [15], the Riemann invariants are chosen with respect to a different entropy level, denoted there as $s_{*}$, which is an upper bound for the values of the entropy (see [15, Lemma 5.1]). Our choice of $\eta_{*}$ is related instead to the lower bound of entropy. As a consequence, the slopes of the shock-rarefaction curves in the $w z$-plane defined below are positive while in [15], they are negative.

The strengths of the shock and rarefaction waves will be measured by $w$ and $z$. The pressure is considered to be a function of $\tau: p=p(\tau)$ and we set

$$
g\left(\tau_{0}, \tau ; \eta_{0}\right)=\mathcal{G}\left(p\left(\tau_{0}\right), p(\tau) ; \eta_{0}\right), \quad h\left(\tau_{0}, \tau\right)=\mathcal{H}\left(p\left(\tau_{0}\right), p(\tau)\right) .
$$

Since $w+z \pm e^{\frac{\epsilon}{\gamma}\left(\eta_{0}-\eta_{*}\right)}(z-w)$ are constant along rarefaction curves, we find that the forward 1-rarefaction curve $\widehat{\mathcal{R}}_{1}^{F}\left(U_{0}\right)$ and the backward 2-rarefaction curve $\widehat{\mathcal{R}}_{2}^{B}\left(U_{0}\right)$ are expressed as

$$
\begin{aligned}
& \widehat{\mathcal{R}}_{1}^{F}\left(U_{0}\right): z-z_{0}=\frac{e^{\frac{\epsilon}{\gamma}\left(\eta_{0}-\eta_{*}\right)}-1}{e^{\frac{\epsilon}{\gamma}\left(\eta_{0}-\eta_{*}\right)}+1}\left(w-w_{0}\right), w \geq w_{0}, \\
& \widehat{\mathcal{R}}_{2}^{B}\left(U_{0}\right): w-w_{0}=\frac{e^{\frac{\epsilon}{\gamma}\left(\eta_{0}-\eta_{*}\right)}-1}{e^{\frac{\epsilon}{\gamma}\left(\eta_{0}-\eta_{*}\right)}+1}\left(z-z_{0}\right), \quad z \leq z_{0} .
\end{aligned}
$$


Note that $0<\frac{e^{\frac{\epsilon}{\gamma}\left(\eta_{0}-\eta_{*}\right)}-1}{e^{\frac{\epsilon}{\gamma}\left(\eta_{0}-\eta_{*}\right)}+1}<1$.

The forward 1-shock curve $\widehat{\mathcal{S}}_{1}^{F}\left(U_{0}\right)$ and the backward 2-shock curve $\widehat{\mathcal{S}}_{2}^{B}\left(U_{0}\right)$ issuing from $U_{0}$ are also represented by using the Riemann invariant coordinates. Remark that by (4.3), the Hugoniot curves through $U_{0}$ are expressed as

$$
u-u_{0}=\mp g\left(\tau_{0}, \tau ; \eta_{0}\right), \quad \eta-\eta_{0}=h\left(\tau_{0}, \tau\right),
$$

which define in particular the function $\eta(\tau)=\eta_{0}+h\left(\tau_{0}, \tau\right)$. Note that $0<\epsilon \leq \frac{1}{3}$ is equivalent to $1<\gamma \leq \frac{5}{3}$.

The following results were proved by Liu [15] (see also Asakura [4]).

Lemma 4.1 If $0<\epsilon \leq \frac{1}{3}$, then there are functions $z_{1}=z_{1}\left(w ; \eta_{0}\right)$ and $w_{2}=w_{2}\left(z ; \eta_{0}\right)$, such that

$$
\begin{aligned}
& \widehat{\mathcal{S}}_{1}^{F}\left(U_{0}\right)=\left\{(w, z, \eta) ; z=z_{1}\left(w ; \eta_{0}\right), \eta=\eta(\tau), w<w_{0}\right\}, \\
& \widehat{\mathcal{S}}_{2}^{B}\left(U_{0}\right)=\left\{(w, z, \eta) ; w=w_{2}\left(z ; \eta_{0}\right), \eta=\eta(\tau), z>z_{0}\right\} .
\end{aligned}
$$

Moreover, there is a constant $B>1$, which depends on $p_{*}, p^{*}, H$, such that

$$
\begin{aligned}
& \frac{e^{\frac{\epsilon}{\gamma}\left(\eta_{0}-\eta_{*}\right)}-1}{e^{\frac{\epsilon}{\gamma}\left(\eta_{0}-\eta_{*}\right)}+1}=z_{1}^{\prime}\left(w_{0} ; \eta_{0}\right) \leq z_{1}^{\prime}\left(w ; \eta_{0}\right) \leq \frac{B e^{\frac{\epsilon}{\gamma}\left(\eta_{0}-\eta_{*}\right)}-1}{B e^{\frac{\epsilon}{\gamma}\left(\eta_{0}-\eta_{*}\right)}+1}, \\
& \frac{e^{\frac{\epsilon}{\gamma}\left(\eta_{0}-\eta_{*}\right)}-1}{e^{\frac{\epsilon}{\gamma}\left(\eta_{0}-\eta_{*}\right)}+1}=w_{2}^{\prime}\left(z_{0} ; \eta_{0}\right) \leq w_{2}^{\prime}\left(z ; \eta_{0}\right) \leq \frac{B e^{\frac{\epsilon}{\gamma}\left(\eta_{0}-\eta_{*}\right)}-1}{B e^{\frac{\epsilon}{\gamma}\left(\eta_{0}-\eta_{*}\right)}+1} .
\end{aligned}
$$

At last

$$
\begin{aligned}
& z_{1}^{\prime \prime}\left(w ; \eta_{0}\right)<0<w_{2}^{\prime \prime}\left(z ; \eta_{0}\right), \\
& \eta_{1}^{\prime}(w)<\eta_{1}^{\prime}\left(w_{0}\right)=0=\eta_{2}^{\prime}\left(z_{0}\right)<\eta_{2}^{\prime}(z) .
\end{aligned}
$$

Lemma 4.2 If $\eta_{1}>\eta_{0}$, then

$$
z_{1}\left(w ; \eta_{1}\right) \leq z_{1}\left(w ; \eta_{0}\right), \quad w_{2}\left(z ; \eta_{1}\right) \leq w_{2}\left(z ; \eta_{0}\right) .
$$

We denote by $\alpha, \beta, \xi, \pi$, respectively, the strengths of 1 -shock waves, 2 -shock waves, 1-rarefaction waves, 2-rarefaction waves, respectively. They are defined by

$$
\begin{aligned}
& \alpha=w_{0}-w \text { if }(p, u, \eta) \in \widehat{\mathcal{S}}_{1}^{F}\left(p_{0}, u_{0}, \eta_{0}\right), \\
& \beta=z-z_{0} \quad \text { if }(p, u, \eta) \in \widehat{\mathcal{S}}_{2}^{B}\left(p_{0}, u_{0}, \eta_{0}\right), \\
& \xi=w-w_{0} \text { if }\left(p, u, \eta_{0}\right) \in \widehat{\mathcal{R}}_{1}^{F}\left(p_{0}, u_{0}, \eta_{0}\right), \\
& \pi=z_{0}-z \quad \text { if }\left(p, u, \eta_{0}\right) \in \widehat{\mathcal{R}}_{2}^{B}\left(p_{0}, u_{0}, \eta_{0}\right) .
\end{aligned}
$$

We also define for entropy waves

$$
\delta=\eta_{L}-\eta_{R} \quad \text { if }\left(p_{0}, u_{0}, \eta_{L}\right),\left(p_{0}, u_{0}, \eta_{R}\right) \text { are the side states. }
$$

The strength of an entropy wave will be defined later at (4.9).

In order to measure the increase in the entropy across shock waves, we define the quantities $\delta_{\alpha}, \delta_{\beta}$ as

$$
\begin{aligned}
& \delta_{\alpha}=\eta-\eta_{0} \text { if }(p, u, \eta) \in \widehat{\mathcal{S}}_{1}^{F}\left(p_{0}, u_{0}, \eta_{0}\right), \\
& \delta_{\beta}=\eta-\eta_{0} \text { if }(p, u, \eta) \in \widehat{\mathcal{S}}_{2}^{B}\left(p_{0}, u_{0}, \eta_{0}\right) .
\end{aligned}
$$

Remark that all these quantities are positive. 
From now on, we also denote by $\alpha, \beta, \delta, \xi, \pi$, the corresponding waves. Suppose that $U_{L}, U_{M}$ are connected by a 2-wave $\theta_{2}$ (or an entropy wave) and $U_{M}, U_{R}$ by a 1-wave $\theta_{1}$ (or an entropy wave); these waves are assumed to interact. Then, under the assumption (2.10), the Riemann problem has a unique solution connecting the states $U_{L}$ and $U_{R}$. It is composed of a 1 -wave $\theta_{1}^{\prime}$ connecting $U_{L}$ to $U^{-}$, an entropy wave $\delta^{\prime}$ connecting $U^{-}$to $U^{+}$and a 2 -wave $\theta_{2}^{\prime}$ connecting $U^{+}$to $U_{R}$. This interaction is simply denoted by

$$
\theta_{2}+\theta_{1} \rightarrow \theta_{1}^{\prime}+\delta^{\prime}+\theta_{2}^{\prime}
$$

The local interaction estimates are gathered in the following lemma, which was first proved by Liu [15]; we also refer to Asakura [4] for more details. Notation below is as in [4] and slightly differs from that in [15].

Lemma 4.3 Assume that $0<\epsilon \leq \frac{1}{3}$. Assume also that $p$ and $\eta$ satisfy (4.1) and $\epsilon H$ is sufficiently small. Then, there are constants $0<D_{0}<1$ and $D, D_{1}, D_{2}>0$, depending only on $p_{*}, p^{*}, H$, such that the following estimates hold.

(1) $\beta+\alpha \rightarrow \alpha^{\prime}+\delta^{\prime}+\beta^{\prime}$; we have $\delta_{\alpha^{\prime}} \geq \delta_{\alpha}-\epsilon D_{2} \alpha \beta, \delta_{\beta^{\prime}} \geq \delta_{\beta}-\epsilon D_{2} \alpha \beta$,

$$
\alpha^{\prime} \leq \alpha+\epsilon D \alpha \beta, \quad\left|\delta^{\prime}\right| \leq \epsilon D_{2} \alpha \beta, \quad \beta^{\prime} \leq \beta+\epsilon D \alpha \beta .
$$

(2) $\pi+\alpha \rightarrow \alpha^{\prime}+\delta^{\prime}+\pi^{\prime}$; we have $\delta_{\alpha^{\prime}} \geq \delta_{\alpha}-\epsilon D_{2} \alpha \pi$,

$$
\alpha^{\prime} \leq \alpha+\epsilon D \alpha \pi, \quad\left|\delta^{\prime}\right| \leq \epsilon D_{2} \alpha \pi, \quad \pi^{\prime} \leq \pi+\epsilon D \alpha \pi .
$$

(3) $\alpha_{1}+\alpha_{2} \rightarrow \alpha^{\prime}+\delta^{\prime}+\pi^{\prime}$; we have $\delta_{\alpha^{\prime}} \geq \delta_{\alpha_{1}}+\delta_{\alpha_{2}}-\epsilon D_{2} \alpha_{1} \alpha_{2}$,

$$
\alpha_{1}+\alpha_{2} \leq \alpha^{\prime} \leq \alpha_{1}+\alpha_{2}+\epsilon D \alpha_{1} \alpha_{2}, \quad\left|\delta^{\prime}\right| \leq D_{2} \alpha_{1} \alpha_{2}, \quad \pi^{\prime} \leq D \alpha_{1} \alpha_{2} .
$$

(4) $\delta+\alpha \rightarrow \alpha^{\prime}+\delta^{\prime}+\theta^{\prime}$; we have $\delta_{\alpha^{\prime}} \geq \delta_{\alpha}-\epsilon D \alpha|\delta|$,

$$
\alpha^{\prime} \leq \alpha+\epsilon D_{1} \alpha|\delta|, \quad\left|\delta^{\prime}\right| \leq|\delta|+\epsilon D \alpha|\delta|, \quad \theta^{\prime} \leq \epsilon D_{1} \alpha|\delta| .
$$

(5) $\delta+\xi \rightarrow \xi^{\prime}+\delta^{\prime}+\theta^{\prime}$; we have

$$
\xi^{\prime} \leq \xi+\epsilon D_{1} \xi|\delta|, \quad\left|\delta^{\prime}\right| \leq|\delta|+\epsilon D \xi|\delta|, \quad \theta^{\prime} \leq \epsilon D_{1} \xi|\delta| .
$$

(6) $\xi+\alpha \rightarrow \alpha^{\prime}+\delta^{\prime}+\beta^{\prime}$; we have $\delta_{\alpha^{\prime}} \geq \delta_{\alpha}-D\left(\alpha-\alpha^{\prime}\right)-\epsilon D_{2} \alpha \xi, \delta_{\beta^{\prime}} \geq 0$,

$$
\alpha^{\prime} \leq \alpha-\xi, \quad\left|\delta^{\prime}\right| \leq D\left(\alpha-\alpha^{\prime}\right)+\epsilon D_{2} \alpha \xi, \quad \beta^{\prime} \leq D_{0}\left(\alpha-\alpha^{\prime}\right)+\epsilon D \alpha^{\prime} \xi .
$$

(7) $\xi+\alpha \rightarrow \xi^{\prime}+\delta^{\prime}+\beta^{\prime}$; we have $\delta_{\beta^{\prime}} \geq \delta_{\alpha}-\epsilon D_{2} \alpha \xi$,

$$
\xi^{\prime} \leq \xi, \quad\left|\delta^{\prime}\right| \leq D \alpha, \quad \beta^{\prime} \leq D_{0} \alpha .
$$

(8) $\alpha+\xi \rightarrow \alpha^{\prime}+\delta^{\prime}+\beta^{\prime}$; we have $\delta_{\alpha^{\prime}} \geq \delta_{\alpha}-D\left(\alpha-\alpha^{\prime}\right)-\epsilon D_{2} \alpha \xi, \delta_{\beta^{\prime}} \geq 0$,

$$
\alpha^{\prime} \leq \alpha-\xi, \quad\left|\delta^{\prime}\right| \leq D\left(\alpha-\alpha^{\prime}\right), \quad \beta^{\prime} \leq D_{0}\left(\alpha-\alpha^{\prime}\right) .
$$

(9) $\alpha+\xi \rightarrow \xi^{\prime}+\delta^{\prime}+\beta^{\prime}$; we have $\delta_{\beta^{\prime}} \geq \delta_{\alpha}-\epsilon D_{2} \alpha \xi$,

$$
\xi^{\prime} \leq \xi, \quad\left|\delta^{\prime}\right| \leq D \alpha, \quad \beta^{\prime} \leq D_{0} \alpha .
$$

(10) $\alpha+\xi \rightarrow \xi^{\prime}+\delta^{\prime}+\pi^{\prime}$; we have

$$
\xi^{\prime} \leq \xi-\alpha, \quad\left|\delta^{\prime}\right|=\delta_{\alpha} \leq D \alpha, \quad \pi^{\prime} \leq \epsilon D \alpha \xi .
$$

(11) $\pi+\xi \rightarrow \xi^{\prime}+\pi^{\prime}$; then $\xi^{\prime}=\xi$ and $\pi^{\prime}=\pi$. 


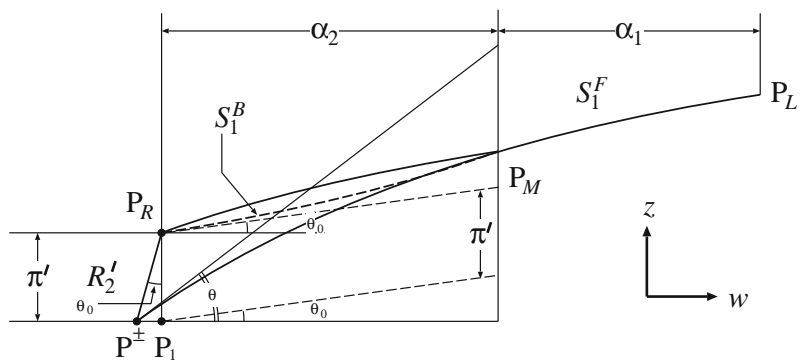

Fig. 1 Proof of the inequality $\pi^{\prime} \leq B_{0} \alpha_{2}$ in Lemma 4.4

Note that

$$
\delta^{\prime}= \begin{cases}\delta_{\alpha^{\prime}}-\delta_{\alpha}-\left(\delta_{\beta^{\prime}}-\delta_{\beta}\right) & \text { in case (1) } \\ \delta_{\alpha^{\prime}}-\delta_{\alpha_{1}}-\delta_{\alpha_{2}} & \text { in case (3). }\end{cases}
$$

Remark also that, because of Remark 4.1, the estimate for $\alpha^{\prime}$ in Case (3) is the converse of that given in [15].

Let us denote by $\mathrm{P}_{L}=\mathrm{P}\left(w_{L}, z_{L}\right), \mathrm{P}_{R}=\mathrm{P}\left(w_{R}, z_{R}\right)$, etc., points of the wz-plane and by $|\mathrm{PQ}|$ the Euclidean distance between two points $\mathrm{P}$ and $\mathrm{Q}$. The projections of the forward and backward shock curves on the $w z$-plane are denoted by $\mathcal{S}_{j}^{F}\left(w_{0}, z_{0} ; \eta_{0}\right)$ and $\mathcal{S}_{j}^{B}\left(w_{0}, z_{0} ; \eta_{0}\right), j=1,2$, respectively.

In addition to Lemma 4.3, we also need the following result.

Lemma 4.4 Consider Case (3) under the assumptions of Lemma 4.3. Then, there is a constant $B_{0}$, which only depends on $p_{*}, p^{*}$ and $H$, such that $0<B_{0}<1$ and

$$
\pi^{\prime} \leq B_{0} \min \left\{\alpha_{1}, \alpha_{2}\right\} .
$$

Proof First, we prove the inequality $\pi^{\prime} \leq B_{0} \alpha_{2}$. Consider the backward 1-shock curve and the backward 2-rarefaction curve issuing from $\mathrm{P}_{R}$ (see Fig. 1). The angle $\theta_{0}$ formed by the tangent to $\mathcal{S}_{1}^{B}\left(w_{R}, z_{R} ; \eta_{R}\right)$ at $\mathrm{P}_{R}$ and the horizontal line equals the one formed by $\mathcal{R}_{2}^{\prime B}=\mathcal{R}_{2}^{B}\left(w_{R}, z_{R} ; \eta_{R}\right)$ and the vertical line $\mathrm{P}_{R} \mathrm{P}_{1}$. Here, $\mathrm{P}_{1}$ is the intersection point $\{z=$ $\left.z_{ \pm}\right\} \cap\left\{w=w_{R}\right\}$. The slope of $\mathcal{S}_{1}^{F}\left(w_{L}, z_{L} ; \eta_{L}\right)$ at $\mathrm{P}_{ \pm}$is denoted by $\tan \theta$.

We have $\left|\mathrm{P}_{ \pm} \mathrm{P}_{1}\right|=\pi^{\prime} \tan \theta_{0}$ and hence

$$
\pi^{\prime} \leq\left(\alpha_{2}+\left|\mathrm{P}_{ \pm} \mathrm{P}_{1}\right|\right) \tan \theta-\alpha_{2} \tan \theta_{0}=\pi^{\prime} \tan \theta \tan \theta_{0}+\alpha_{2}\left(\tan \theta-\tan \theta_{0}\right) .
$$

Thus, we have

$$
\pi^{\prime} \leq \frac{\alpha_{2}\left(\tan \theta-\tan \theta_{0}\right)}{1-\tan \theta \tan \theta_{0}} .
$$

By Lemma 4.1 and (4.1), we deduce

$$
0<\tan \theta \leq \frac{B e^{\frac{\epsilon}{\gamma} H}-1}{B e^{\frac{\epsilon}{\gamma} H}+1}<1 .
$$

Moreover, for $\theta$ fixed, the function $\phi(\xi)=\frac{\tan \theta-\xi}{1-(\tan \theta) \xi}$ is a decreasing function of $\xi$ in $[0, \tan \theta]$. Then, by (4.7), we conclude that

$$
\pi^{\prime} \leq \alpha_{2} \tan \theta \leq \frac{B e^{\frac{\epsilon}{\gamma} H}-1}{B e^{\frac{\epsilon}{\gamma} H}+1} \alpha_{2}=: B_{0} \alpha_{2},
$$




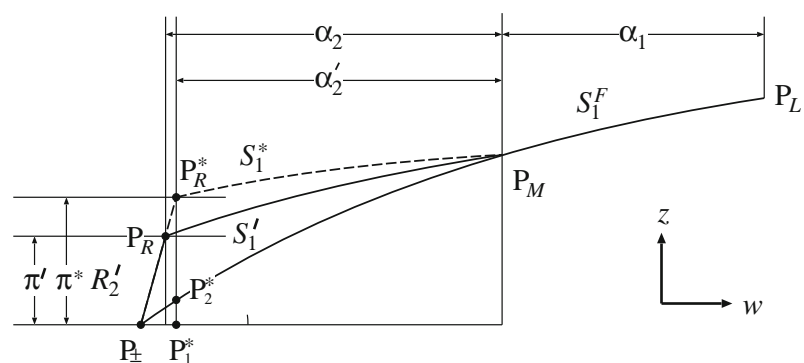

Fig. 2 Proof of the inequality $\pi^{\prime} \leq B_{0} \alpha_{1}$ in Lemma 4.4

and we have proved the desired inequality.

Now, we prove the inequality $\pi^{\prime} \leq B_{0} \alpha_{1}$; we refer to Fig. 2 . We first notice that $\eta_{M}>\eta_{L}$; hence, by Lemma 4.2, we have

$$
z_{1}\left(w ; \eta_{M}\right)<z_{1}\left(w ; \eta_{L}\right)
$$

This shows that the shock curve $\mathcal{S}_{1}^{*}=\mathcal{S}_{1}^{F}\left(w_{M}, z_{M} ; \eta_{L}\right)$ is located above the curve $\mathcal{S}_{1}^{\prime}=$ $\mathcal{S}_{1}^{F}\left(w_{M}, z_{M} ; \eta_{M}\right)$. Hence, we have

$$
\begin{aligned}
\pi^{*} & =\left|\mathrm{P}_{R}^{*} \mathrm{P}_{2}^{*}\right|+\left|\mathrm{P}_{2}^{*} \mathrm{P}_{1}^{*}\right| \leq\left|\mathrm{P}_{R}^{*} \mathrm{P}_{2}^{*}\right|+\frac{B e^{\frac{\epsilon}{\gamma} H}-1}{B e^{\frac{\epsilon}{\gamma} H}+1}\left|\mathrm{P}_{ \pm} \mathrm{P}_{1}^{*}\right| \\
& \leq\left|\mathrm{P}_{R}^{*} \mathrm{P}_{2}^{*}\right|+\frac{B e^{\frac{\epsilon}{\gamma} H}-1}{B e^{\frac{\epsilon}{\gamma} H}+1} \cdot \frac{e^{\frac{\epsilon}{\gamma} H}-1}{e^{\frac{\epsilon}{\gamma} H}+1} \cdot \pi^{*} .
\end{aligned}
$$

Thus, we obtain

$$
\pi^{\prime}<\pi^{*} \leq \frac{\left(B e^{\frac{\epsilon}{\gamma} H}+1\right)\left(e^{\frac{\epsilon}{\gamma} H}+1\right)}{2(B+1) e^{\frac{\epsilon}{\gamma} H}} \cdot\left|\mathrm{P}_{R}^{*} \mathrm{P}_{2}^{*}\right| .
$$

Since $\pi^{\prime \prime}=\left|\mathrm{P}_{R}^{*} \mathrm{P}_{2}^{*}\right|$ is the strength of the rarefaction wave generated by the interaction

$$
\alpha_{1}+\alpha_{2}^{\prime} \rightarrow \alpha^{\prime \prime}+\pi^{\prime \prime} \quad\left(\alpha^{\prime \prime}=\alpha_{1}+\alpha_{2}^{\prime}\right)
$$

in the isentropic gas dynamics, we find some $0<D_{0}^{\prime}<1$ so that $\pi^{\prime \prime} \leq D_{0}^{\prime} \alpha_{1}$, [18]. With a slight abuse of notation set

$$
B_{0}:=\frac{\left(B e^{\frac{\epsilon}{\gamma} H}+1\right)\left(e^{\frac{\epsilon}{\gamma} H}+1\right)}{2(B+1) e^{\frac{\epsilon}{\gamma} H}} D_{0}^{\prime} .
$$

Then, $B_{0}<1$ if $\epsilon H$ sufficiently small ${ }^{1}$ and we obtain the desired estimate.

In Lemma 4.4, the assumption $0<\epsilon \leq \frac{1}{3}$ and the smallness of $\epsilon H$ were only used for the inequality $\pi^{\prime} \leq B_{0} \alpha_{1}$ and not for the inequality $\pi^{\prime} \leq B_{0} \alpha_{2}$.

Remark 4.2 We refer to the cases listed in Lemma 4.3. In Cases (1)-(5), the waves outgoing from an interaction are estimated in a quadratic way by $\epsilon$ times the product of the incoming waves, namely by $\epsilon D \alpha \beta, \epsilon D \alpha \pi, \epsilon D \alpha_{1} \alpha_{2}, \epsilon \alpha|\delta|, \epsilon D \xi|\delta|$, respectively. An exception is the

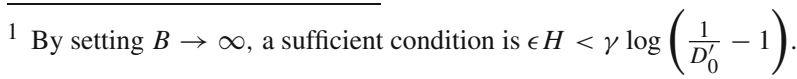


case of $\delta^{\prime}$ and $\pi^{\prime}$ in Case (3), where $\epsilon$ is missing. This case requires a special consideration below; in particular for $\pi^{\prime}$, we shall use Lemma 4.4. The interaction estimates in Cases (6)-(10) are of a different nature and are considered separately.

Therefore, the local interactions are subdivided into the following 4 groups:

$$
\begin{array}{llll}
A: & (1),(2),(11) ; & B: & (3) ; \\
C: & (4),(5) ; & D: & (6),(7),(8),(9),(10) .
\end{array}
$$

Since the estimates are similar within each group, we shall discuss only Cases (1), (3), (4), and (6) as typical ones.

Now, we introduce a small number $M_{0}>0$ to be fixed later on at (5.28) and define the strength of an entropy wave $\delta$ as

$$
M_{0}|\delta|
$$

This definition aims at controlling the outgoing waves, as we now show.

- Consider Case (3), by Lemma 4.4, we have

$$
\begin{aligned}
\pi^{\prime}+M_{0}\left|\delta^{\prime}\right| & \leq B_{0} \min \left\{\alpha_{1}, \alpha_{2}\right\}+M_{0} D_{2} \alpha_{1} \alpha_{2} \\
& \leq\left\{B_{0}+M_{0} D_{2} \sup _{\alpha} \alpha\right\} \min \left\{\alpha_{1}, \alpha_{2}\right\} .
\end{aligned}
$$

Then, the number $M_{0}$ is chosen so small that

$$
B_{0}+M_{0} D_{2} \sup _{\alpha} \alpha \leq D_{*}<1,
$$

where $D_{*} \in\left(D_{0}, 1\right)$ is an arbitrary number fixed once for all [we warn the reader that, for typographical reasons, from Sect. 7 on, we shall denote $D_{*}=c$, see (7.3)]. The requirement that $D_{*}>D_{0}$ is going to be used in (4.11) and (4.15).

- Consider Case (6) and assume $D_{2} M_{0}<D$, see (5.14). Since $\alpha^{\prime} \leq \alpha-\xi$, we find that

$$
\begin{aligned}
\beta^{\prime}+M_{0}\left|\delta^{\prime}\right| & \leq\left(D_{0}+D M_{0}\right)\left(\alpha-\alpha^{\prime}\right)+\epsilon\left(D \alpha^{\prime}+D_{2} M_{0} \alpha\right) \xi \\
& \leq\left\{D_{0}+D M_{0}+2 \epsilon D \alpha\right\}\left(\alpha-\alpha^{\prime}\right) .
\end{aligned}
$$

Let $\epsilon \sup \alpha$ and $M_{0}$ be so small that

$$
D_{0}+D M_{0}+2 \epsilon D \sup _{\alpha} \alpha \leq D_{*}<1 .
$$

Then, we have the following important estimate:

$$
\beta^{\prime}+M_{0}\left|\delta^{\prime}\right| \leq D_{*}\left(\alpha-\alpha^{\prime}\right) .
$$

Moreover, unless of replacing $D$ with $D+\epsilon D_{2} \sup _{\alpha} \alpha$, we may assume $\delta_{\alpha^{\prime}} \geq \delta_{\alpha}-D(\alpha-$ $\alpha^{\prime}$ ). We denote by $C_{H}$ a strictly positive constant, which depends on $p_{*}, p^{*}$ and $H$, such that $\delta_{\alpha} \leq C_{H} \alpha$ and analogously for $\beta$. We also choose $M_{0}$ so small that

$$
M_{0} C_{H} \leq \frac{1}{2} \text {. }
$$

In Cases (7), (9), and (10), the quantity $\alpha^{\prime}$ in (4.12) is missing; moreover, in Case (10), $\beta^{\prime}$ is replaced by $\pi^{\prime}$. If we denote both $\beta^{\prime}$ and $\pi^{\prime}$ with $\theta^{\prime}$, then we easily see that the estimate

$$
\theta^{\prime}+M_{0}\left|\delta^{\prime}\right| \leq D_{*} \alpha,
$$

holds for all Cases (6)-(10), provided that (4.11) is replaced by

$$
D_{0}+D M_{0}+2 \epsilon D \sup _{\alpha, \beta, \xi, \pi}\{\alpha, \beta, \xi, \pi\} \leq D_{*}<1 .
$$




\section{Global interaction estimates}

We consider an approximate solution $U^{h}=\left(p^{h}, u^{h}, \eta^{h}\right)$ defined for $t \in[0, T]$ as in Sect. 3; for simplicity, we often drop the superscript $h$. Then, we denote $w(x, t)=$ $w(p(x, t), u(x, t)), z(x, t)=z(p(x, t), u(x, t))$ and $\bar{w}(x)=w(x, 0), \bar{z}(x)=z(x, 0)$.

Let $\mathrm{P}, \mathrm{Q}$ be two arbitrary points in the $w z$-plane. By denoting $w_{1}=z, w_{2}=w$, we define a distance in that plane by

$$
|\mathrm{P}-\mathrm{Q}|=\max _{j=1,2}\left\{\left|w_{j}(\mathrm{P})-w_{j}(\mathrm{Q})\right|\right\} .
$$

Since $\operatorname{TV}(\bar{p}(x), \bar{u}(x), \bar{\eta}(x))$ is finite, there are limit states

$$
U_{ \pm \infty}=\left(p_{ \pm \infty}, u_{ \pm \infty}, \eta_{ \pm \infty}\right)=\lim _{x \rightarrow \pm \infty}(\bar{p}(x), \bar{u}(x), \bar{\eta}(x))
$$

whose projections on the $w z$-plane are simply denoted by $\mathrm{P}_{ \pm \infty}=\mathrm{P}\left(w_{ \pm \infty}, z_{ \pm \infty}\right)$. Consider a sequence of states $U_{-\infty}=U_{0}, U_{1}, U_{2}, \ldots, U_{n}=U_{\infty}$, connected by $j$-waves $(1 \leq j \leq 2)$ and denote by $\mathrm{P}_{-\infty}=\mathrm{P}_{0}, \mathrm{P}_{1}, \mathrm{P}_{2}, \ldots, \mathrm{P}_{n}=\mathrm{P}_{\infty}$, their projections in the $w z$-plane. We define

$$
\begin{aligned}
& \mathcal{L}_{j}^{+}=\left\{l: w_{j}\left(\mathrm{P}_{l}\right) \geq w_{j}\left(\mathrm{P}_{l-1}\right), 1 \leq l \leq n\right\}, \\
& \mathcal{L}_{j}^{-}=\left\{l: w_{j}\left(\mathrm{P}_{l}\right)<w_{j}\left(\mathrm{P}_{l-1}\right), 1 \leq l \leq n\right\}
\end{aligned}
$$

and then

$$
\mathcal{L}^{-}=\left\{l: U_{l-1} \text { and } U_{l} \text { are connected by a shock wave, } 1 \leq l \leq n\right\} .
$$

Then, we have (see Asakura and Corli [5], Theorem A.4)

$$
\sum_{1 \leq l \leq n}\left|\mathrm{P}_{l}-\mathrm{P}_{l-1}\right| \leq 2\left|\mathrm{P}_{\infty}-\mathrm{P}_{-\infty}\right|+3 \sum_{l \in \mathcal{L}^{-}}\left|\mathrm{P}_{l}-\mathrm{P}_{l-1}\right| .
$$

For any space-like curve $J$ containing no interaction points (this condition is assumed in the following for any space-like curve, without any further mention), we define the global interaction functional $F$ by

$$
\begin{aligned}
F(J)= & L_{F}(J)+\epsilon Q(J), \\
L_{F}(J)= & \sum_{J}\left\{\left(\alpha-M_{0} \delta_{\alpha}\right)+\left(\beta-M_{0} \delta_{\beta}\right)+M_{0}|\delta|\right\}, \\
Q(J)= & M_{0} M_{1} \sum_{J: \mathcal{A}}(\alpha+\beta+\xi+\pi)|\delta| \\
& +M_{1} \sum_{J: \mathcal{A}}(\xi \alpha+\xi \beta+\pi \alpha+\pi \beta)+M_{2} \sum_{J: \mathcal{A}}\left(\alpha_{1} \alpha_{2}+\alpha \beta+\beta_{1} \beta_{2}\right) .
\end{aligned}
$$

Here, $M_{1}$ and $M_{2}$ are positive parameters to be fixed later on. Moreover, $\sum_{J}\left(\sum_{J: \mathcal{A}}\right)$ denotes the summation of all waves crossing $J$ (and approaching, respectively, [7]). We also define

$$
G(J)=2\left|\mathrm{P}_{\infty}-\mathrm{P}_{-\infty}\right|+8 F(J) .
$$

Above, we defined the functionals in terms of space-like curves, instead of $t$, in view of further applications, see [12], [7, §7.5]. We denote

$$
\begin{aligned}
\operatorname{TV}(J) & =T V_{D}(J)+M_{0} T V_{H}(J), \\
T V_{D}(J) & =\left.\operatorname{TV}(w(x, t), z(x, t))\right|_{(x, t) \in J}, \\
T V_{H}(J) & =\left.\operatorname{TV} \eta(x, t)\right|_{(x, t) \in J},
\end{aligned}
$$


where $T V_{D}(J)$ is computed with respect to the metric (5.1). We also define

$$
M_{3}=M_{1}+M_{2} \text {. }
$$

Proposition 5.1 If the constants $M_{0}, M_{1}, M_{2}$ satisfy (4.13) and

$$
\epsilon M_{3} G(J) \leq 1,
$$

then

$$
\frac{1}{2} F(J) \leq \mathrm{TV}(J) \leq G(J)
$$

Proof By (4.13), we deduce

$$
\alpha-M_{0} \delta_{\alpha} \geq \alpha / 2, \quad \beta-M_{0} \delta_{\beta} \geq \beta / 2 .
$$

Then, by (5.2),

$$
\begin{aligned}
\operatorname{TV}(J) & \leq 2\left|\mathrm{P}_{\infty}-\mathrm{P}_{-\infty}\right|+\left(3+C_{H} M_{0}\right) \sum_{J}(\alpha+\beta)+M_{0} \sum_{J}|\delta| \\
& \leq 2\left|\mathrm{P}_{\infty}-\mathrm{P}_{-\infty}\right|+8 L_{F}(J),
\end{aligned}
$$

that proves the inequality on the right of (5.10); remark that condition (5.9) has not been used. By this inequality and (5.9), we have

$$
\begin{aligned}
F(J) \leq & T V_{D}(J)+M_{0} T V_{H}(J) \\
& +\epsilon M_{0} M_{1} T V_{D}(J) T V_{H}(J)+\epsilon M_{3} T V_{D}(J)^{2} \\
\leq & \operatorname{TV}(J)+\epsilon M_{3} G(J) \operatorname{TV}(J) \leq 2 \operatorname{TV}(J),
\end{aligned}
$$

that proves the inequality on the left of (5.10).

Let $O$ be a space-like curve such that there are no points of interaction between $O$ and $t=0$. The global interaction estimates consist in showing

$$
F(J) \leq F(O),
$$

for any arbitrary space-like curve $J$, provided that $\epsilon \operatorname{TV}(O)$ is sufficiently small and $M_{0}, M_{1}, M_{2}$ satisfy a set of constraints. We assume, analogously to (4.1),

$$
G(O) \leq G^{*},
$$

for some fixed constant $G^{*}$ (defined in the proof of Lemma (5.2)). About $M_{0}$, besides the conditions (4.11), (4.13), we further require that

$$
M_{0} D \leq D_{1}, \quad M_{0} D_{2} \leq D .
$$

To prove (5.12), we argue as follows. Suppose that (5.12) holds for $J$ and for every spacelike curve lying between $J$ and $t=0$. Let $J^{\prime}$ be a space-like curve lying between $J$ and $t=\infty$. If there are no interaction points between $J$ and $J^{\prime}$, then obviously $F\left(J^{\prime}\right)=F(J) \leq F(O)$. Let us assume that there is a single interaction point $\mathrm{P}$ between $J^{\prime}$ and $J$.

If $\theta^{\prime}, \theta^{\prime \prime}$ are the two incoming waves at $\mathrm{P}$, we define

$$
Q(\mathrm{P})= \begin{cases}D \theta^{\prime} \theta^{\prime \prime} & \text { if both } \theta^{\prime} \text { and } \theta^{\prime \prime} \text { are } 1 \text { or } 2 \text { waves, } \\ D_{1}\left|\theta^{\prime} \theta^{\prime \prime}\right| & \text { if either } \theta^{\prime} \text { or } \theta^{\prime \prime} \text { is an entropy wave. }\end{cases}
$$

We shall show a stronger estimate, which is needed later on (Lemma 5.3),

$$
F\left(J^{\prime}\right)-F(J) \leq-3 \epsilon Q(\mathrm{P}),
$$


for any case of Lemma 4.3. Then, by an inductive argument, we have (5.12) for all $J$.

According to the cases that we are dealing with, we impose some conditions on the parameters; Lemma 5.2 shall prove that a choice of the parameters can be done once for all. As we mentioned in Remark 4.2, we shall carry out the estimates only for the most complicated cases (1), (3), (4), and (6). Below, we use Lemma 4.3 several times.

Case (1): $\beta+\alpha \rightarrow \alpha^{\prime}+\delta^{\prime}+\beta^{\prime}$. We have

$$
\begin{aligned}
L_{F}\left(J^{\prime}\right)-L_{F}(J) & =\left(\alpha^{\prime}-\alpha\right)-M_{0}\left(\delta_{\alpha^{\prime}}-\delta_{\alpha}\right)+\left(\beta^{\prime}-\beta\right)-M_{0}\left(\delta_{\beta^{\prime}}-\delta_{\beta}\right)+M_{0}\left|\delta^{\prime}\right| \\
& \leq 2 \epsilon D \alpha \beta+M_{0}\left\{\left|\delta^{\prime}\right|-\left(\delta_{\alpha^{\prime}}-\delta_{\alpha}\right)-\left(\delta_{\beta^{\prime}}-\delta_{\beta}\right)\right\} .
\end{aligned}
$$

By (4.5), if $\delta^{\prime} \geq 0$ we have $\left|\delta^{\prime}\right|=\delta_{\alpha^{\prime}}-\delta_{\alpha}-\left(\delta_{\beta^{\prime}}-\delta_{\beta}\right)$; if $\delta^{\prime}<0$, we have $\left|\delta^{\prime}\right|=$ $\delta_{\beta^{\prime}}-\delta_{\beta}-\left(\delta_{\alpha^{\prime}}-\delta_{\alpha}\right)$. In both cases by (5.14) we obtain

$$
L_{F}\left(J^{\prime}\right)-L_{F}(J) \leq 4 \epsilon D \alpha \beta
$$

Moreover, by (5.10) and (5.14), we have

$$
\begin{aligned}
Q\left(J^{\prime}\right)-Q(J) \leq & M_{0} M_{1}\left(\sum\left(\alpha_{J}+\beta_{J}+\xi_{J}+\pi_{J}\right)\left|\delta^{\prime}\right|+\sum\left(\left[\alpha^{\prime}-\alpha\right]_{+}+\left[\beta^{\prime}-\beta\right]_{+}\right)\left|\delta_{J}\right|\right) \\
& +M_{1} \sum\left(\xi_{J}+\pi_{J}\right)\left(\left[\alpha^{\prime}-\alpha\right]_{+}+\left[\beta^{\prime}-\beta\right]_{+}\right) \\
& +M_{2} \sum\left(\alpha_{J}\left[\alpha^{\prime}-\alpha\right]_{+}+\beta_{J}\left[\beta^{\prime}-\beta\right]_{+}\right)-M_{2} \alpha \beta \\
\leq & M_{0} M_{1} T V_{D}(J) \epsilon D_{2} \alpha \beta+2 M_{0} M_{1} T V_{H}(J) \epsilon D \alpha \beta+2 M_{1} T V_{D}(J) \epsilon D \alpha \beta \\
& +2 M_{2} T V_{D}(J) \epsilon D \alpha \beta-M_{2} \alpha \beta \\
\leq & \left\{3 \epsilon D M_{3} G(J)-M_{2}\right\} \alpha \beta .
\end{aligned}
$$

Here, $\sum\left(\alpha_{J}+\beta_{J}\right)$ denotes the total amount of shock waves crossing $J$ and so on; we used the notation $[x]_{+}=\max \{x, 0\}$. Hence, by $(5.12)$ and assuming

$$
7 D+3 \epsilon D M_{3} G(O) \leq M_{2}
$$

we have $Q\left(J^{\prime}\right)-Q(J) \leq-7 D \alpha \beta$. Then, (5.16) follows.

Case (3): $\alpha_{1}+\alpha_{2} \rightarrow \alpha^{\prime}+\delta^{\prime}+\pi^{\prime}$. We have

$$
\begin{aligned}
L_{F}\left(J^{\prime}\right)-L_{F}(J) & =\left(\alpha^{\prime}-\alpha_{1}-\alpha_{2}\right)-M_{0}\left(\delta_{\alpha^{\prime}}-\delta_{\alpha_{1}}-\delta_{\alpha_{2}}\right)+M_{0}\left|\delta^{\prime}\right| \\
& \leq \epsilon D \alpha_{1} \alpha_{2}+M_{0}\left\{\left|\delta^{\prime}\right|-\left(\delta_{\alpha^{\prime}}-\delta_{\alpha_{1}}-\delta_{\alpha_{2}}\right)\right\} .
\end{aligned}
$$

By (4.5) and (5.14), if $\delta_{\alpha^{\prime}} \geq \delta_{\alpha_{1}}+\delta_{\alpha_{2}}$, we have $\left|\delta^{\prime}\right|=\delta_{\alpha^{\prime}}-\delta_{\alpha_{1}}-\delta_{\alpha_{2}}$ and $L_{F}\left(J^{\prime}\right)-L_{F}(J) \leq$ $\epsilon D \alpha_{1} \alpha_{2}$. If $\delta_{\alpha^{\prime}}<\delta_{\alpha_{1}}+\delta_{\alpha_{2}}$, we have $\left|\delta^{\prime}\right|=\delta_{\alpha_{1}}+\delta_{\alpha_{2}}-\delta_{\alpha^{\prime}} \leq \epsilon D_{2} \alpha_{1} \alpha_{2}$ and hence $L_{F}\left(J^{\prime}\right)-L_{F}(J) \leq 3 \epsilon D \alpha_{1} \alpha_{2}$ by (5.14). In any case, we have

$$
L_{F}\left(J^{\prime}\right)-L_{F}(J) \leq 3 \epsilon D \alpha_{1} \alpha_{2}
$$


We find by (5.14)

$$
\begin{aligned}
Q\left(J^{\prime}\right)-Q(J) \leq & M_{0} M_{1}\left(\sum\left(\alpha_{J}+\beta_{J}+\xi_{J}+\pi_{J}\right)\left|\delta^{\prime}\right|+\sum\left(\left[\alpha^{\prime}-\alpha_{1}-\alpha_{2}\right]_{+}+\pi^{\prime}\right)\left|\delta_{J}\right|\right) \\
& +M_{1} \sum\left(\alpha_{J}+\beta_{J}\right) \pi^{\prime}+M_{1} \sum\left(\xi_{J}+\pi_{J}\right)\left[\alpha^{\prime}-\alpha_{1}-\alpha_{2}\right]_{+} \\
& +M_{2} \sum\left(\alpha_{J}+\beta_{J}\right)\left[\alpha^{\prime}-\alpha_{1}-\alpha_{2}\right]_{+}-M_{2} \alpha_{1} \alpha_{2} \\
\leq & M_{0} M_{1} T V_{D}(J) D_{2} \alpha_{1} \alpha_{2}+(\epsilon+1) M_{0} M_{1} T V_{H}(J) D \alpha_{1} \alpha_{2} \\
& +M_{1} T V_{D}(J) D \alpha_{1} \alpha_{2}+M_{1} T V_{D}(J) \epsilon D \alpha_{1} \alpha_{2} \\
& +M_{2} T V_{D}(J) \epsilon D \alpha_{1} \alpha_{2}-M_{2} \alpha_{1} \alpha_{2} \\
\leq & \left\{2 D M_{1} G(J)+\epsilon D M_{3} G(J)-M_{2}\right\} \alpha_{1} \alpha_{2} .
\end{aligned}
$$

Hence, by the hypothesis (5.12) and assuming

$$
6 D+D\left\{2 M_{1}+\epsilon M_{3}\right\} G(O) \leq M_{2},
$$

we obtain $Q\left(J^{\prime}\right)-Q(J) \leq-6 D \alpha_{1} \alpha_{2}$. As a consequence, (5.16) follows.

Case (4): $\delta+\alpha \rightarrow \alpha^{\prime}+\delta^{\prime}+\theta^{\prime}$. First, letting $\delta_{\pi^{\prime}}=0$,

$$
\begin{aligned}
L_{F}\left(J^{\prime}\right)-L_{F}(J) & =\left(\alpha^{\prime}-\alpha\right)-M_{0}\left(\delta_{\alpha^{\prime}}-\delta_{\alpha}\right)+\theta^{\prime}-M_{0} \delta_{\theta^{\prime}}+M_{0}\left(\left|\delta^{\prime}\right|-|\delta|\right) \\
& \leq 3 \epsilon D_{1}|\delta| \alpha+M_{0} C_{H} \epsilon D|\delta| \alpha+M_{0} \epsilon D|\delta| \alpha \\
& \leq 5 \epsilon D_{1}|\delta| \alpha,
\end{aligned}
$$

by (4.13). Second,

$$
\begin{aligned}
Q\left(J^{\prime}\right)-Q(J) \leq & M_{0} M_{1}\left(\sum\left(\alpha_{J}+\beta_{J}+\xi_{J}+\pi_{J}\right)\left[\left|\delta^{\prime}\right|-|\delta|\right]_{+}+\sum\left(\left[\alpha^{\prime}-\alpha\right]_{+}+\theta^{\prime}\right)\left|\delta_{J}\right|\right) \\
& -M_{0} M_{1}|\delta| \alpha+M_{1} \sum\left(\alpha_{J}+\beta_{J}+\xi_{J}+\pi_{J}\right) \theta^{\prime} \\
& +M_{2} \sum\left(\alpha_{J}+\beta_{J}\right)\left(\left[\alpha^{\prime}-\alpha\right]_{+}+\theta^{\prime}\right) \\
\leq & M_{0} M_{1} T V_{D}(J) \epsilon D|\delta| \alpha+2 M_{0} M_{1} T V_{H}(J) \epsilon D_{1}|\delta| \alpha-M_{0} M_{1}|\delta| \alpha \\
& +M_{1} T V_{D}(J) \epsilon D_{1}|\delta| \alpha+2 M_{2} T V_{D}(J) \epsilon D_{1}|\delta| \alpha \\
\leq & \left\{2 \epsilon D_{1} M_{3} G(J)-M_{0} M_{1}\right\}|\delta| \alpha .
\end{aligned}
$$

Hence, by using the hypothesis (5.12) and assuming

$$
8 D_{1}+2 \epsilon D_{1} M_{3} G(O) \leq M_{0} M_{1},
$$

we obtain $Q\left(J^{\prime}\right)-Q(J) \leq-8 D_{1}|\delta| \alpha$. Then, (5.16) follows.

Case (6): $\xi+\alpha \rightarrow \alpha^{\prime}+\delta^{\prime}+\beta^{\prime}$. We have

$$
L_{F}\left(J^{\prime}\right)-L_{F}(J)=\left(\alpha^{\prime}-\alpha\right)-M_{0}\left(\delta_{\alpha^{\prime}}-\delta_{\alpha}\right)+\beta^{\prime}-M_{0} \delta_{\beta^{\prime}}+M_{0}\left|\delta^{\prime}\right| .
$$

Note that, by (5.11),

$$
\begin{aligned}
\alpha^{\prime}-\alpha+\beta^{\prime} & \leq-\left(1-D_{0}\right)\left(\alpha-\alpha^{\prime}\right)+\epsilon D \alpha \xi \\
& \leq-\left\{1-D_{0}-2 \epsilon F(J) D\right\}\left(\alpha-\alpha^{\prime}\right) .
\end{aligned}
$$

We conclude that

$$
L_{F}\left(J^{\prime}\right)-L_{F}(J)=-\left\{1-D_{0}-2 M_{0} D-2 D \epsilon F(J)\right\}\left(\alpha-\alpha^{\prime}\right)+2 \epsilon M_{0} D_{2} \alpha \xi .
$$


Moreover,

$$
\begin{aligned}
Q\left(J^{\prime}\right)-Q(J) \leq & M_{0} M_{1}\left(\sum\left(\alpha_{J}+\beta_{J}+\xi_{J}+\pi_{J}\right)\left|\delta^{\prime}\right|+\sum \beta^{\prime}\left|\delta_{J}\right|\right) \\
& +M_{1} \sum\left(\xi_{J}+\pi_{J}\right) \beta^{\prime}-M_{1} \alpha \xi+M_{2} \sum\left(\alpha_{J}+\beta_{J}\right) \beta^{\prime} \\
\leq & M_{0} M_{1} T V_{D}(J)\left|\delta^{\prime}\right|+M_{0} M_{1} \beta^{\prime} T V_{H}(J)+M_{1} \beta^{\prime} T V_{D}(J) \\
& -M_{1} \alpha \xi+M_{2} T V_{D}(J) \beta^{\prime} \\
\leq & M_{0} M_{1} G(J)\left|\delta^{\prime}\right|+M_{3} G(J) \beta^{\prime}-M_{1} \alpha \xi \\
\leq & \left(2 M_{1}+M_{2}\right) D_{1} G(J)\left(\alpha-\alpha^{\prime}\right)+\left\{\epsilon\left(2 M_{1}+M_{2}\right) D G(J)-M_{1}\right\} \alpha \xi .
\end{aligned}
$$

Remark that we may replace the constants $D, D_{1}, D_{2}$ in Lemma 4.3 by larger ones: the result still holds. Then, we assume $D_{1} \geq D_{0}$; by (5.12), (5.14), we obtain

$$
\begin{aligned}
& F\left(J^{\prime}\right)-F(J) \\
& \quad \leq-\left\{1-D_{0}-2 M_{0} D-2 \epsilon D F(O)-\epsilon^{2}\left(2 M_{1}+M_{2}\right) D_{1} G(O)\right\}\left(\alpha-\alpha^{\prime}\right) \\
& \quad+\epsilon\left\{\epsilon\left(2 M_{1}+M_{2}\right) D G(O)-\left(M_{1}-2 M_{0} D_{2}\right)\right\} \alpha \xi .
\end{aligned}
$$

By assuming

$$
\begin{aligned}
2 \epsilon D F(O)+\epsilon^{2}\left(2 M_{1}+M_{2}\right) D_{1} G(O) & \leq 1-D_{0}-2 M_{0} D, \\
\epsilon\left(2 M_{1}+M_{2}\right) D G(O) & \leq M_{1}-2 M_{0} D_{2}-3 D,
\end{aligned}
$$

we obtain (5.16) also in this case.

Lemma 5.1 Consider any approximate solution $U^{h}$, define

$$
k=\frac{1}{2} \sqrt{\gamma} a^{\frac{1}{\gamma}} e^{\frac{\epsilon}{\gamma} \eta_{*}} p_{-\infty}^{\frac{\epsilon}{\gamma}}
$$

and assume that for some constant $K>0$ and any $J$, we have

$$
T V_{D}(J) \leq K, \quad \epsilon T V_{D}(J) \leq k .
$$

Then, there exists $p_{*}, p^{*}$, and $H$, which depend on the initial data (3.1) and on $K$, such that $U^{h}$ is valued in the region (4.1).

Proof By (4.2), we have

$$
\frac{\left(p^{h}\right)^{\frac{\epsilon}{\gamma}}-\bar{p}_{*}^{\frac{\epsilon}{\gamma}}}{\epsilon}=\frac{z^{h}-w^{h}}{2 \sqrt{\gamma} a^{\frac{1}{\gamma}} e^{\frac{\epsilon}{\gamma} \eta_{*}}} .
$$

By (5.10), we deduce that, for any $t$,

$$
\operatorname{TV}\left[\frac{\left(p^{h}\right)^{\frac{\epsilon}{\gamma}}-\bar{p}_{*}^{\frac{\epsilon}{\gamma}}}{\epsilon}\right] \leq \frac{\operatorname{TV}_{D}(t)}{\sqrt{\gamma} a^{\frac{1}{\gamma}} e^{\frac{\epsilon}{\gamma} \eta_{*}}},
$$

where the total variation on the left-hand side is the usual total variation with respect to the variable $x$ and $\operatorname{TV}_{D}(t)$ is referred to the horizontal space-like curve at time $t$. Then,

$$
\left|\frac{\left(p^{h}\right)^{\frac{\epsilon}{\gamma}}-p_{-\infty}^{\frac{\epsilon}{\gamma}}}{\epsilon}\right|=\left|\frac{\left(p^{h}\right)^{\frac{\epsilon}{\gamma}}-\bar{p}_{*}^{\frac{\epsilon}{\gamma}}}{\epsilon}-\frac{p_{-\infty}^{\frac{\epsilon}{\gamma}}-\bar{p}_{*}^{\frac{\epsilon}{\gamma}}}{\epsilon}\right| \leq \frac{\operatorname{TV}_{D}(t)}{\sqrt{\gamma} a^{\frac{1}{\gamma}} e^{\frac{\epsilon}{\gamma} \eta_{*}}} .
$$


As a consequence,

$$
\frac{\left|\left(\frac{p^{h}}{p_{-\infty}}\right)^{\frac{\epsilon}{\gamma}}-1\right|}{\epsilon} \leq \frac{K}{2 k}
$$

We first look for the upper bound $p^{*}$ of $p^{h}$. If $p^{h} \leq p_{-\infty}$, we are done. Otherwise, let $E^{*}$ be the set of points $(t, x)$ where $p^{h}(t, x)>p_{-\infty}$ and only consider points in $E^{*}$. By (5.26) and introducing the increasing function $\phi(y)=\left(e^{y}-1\right) / y$, we deduce

$$
\gamma \log \frac{p^{h}}{p_{-\infty}}=\gamma \log \frac{p^{h}}{p_{-\infty}} \phi(0) \leq \gamma \log \frac{p^{h}}{p_{-\infty}} \phi\left(\frac{\epsilon}{\gamma} \log \frac{p^{h}}{p_{-\infty}}\right) \leq \frac{K}{2 k},
$$

whence

$$
p^{h} \leq p_{-\infty} e^{K /(2 \gamma k)} \doteq p^{*} .
$$

We now look for a lower bound $p_{*}$ of $p^{h}$ by arguing as above. If $p^{h} \geq p_{-\infty}$, we are done. Otherwise, let $E_{*}$ be the set of points $(t, x)$ where $p^{h}(t, x)<p_{-\infty}$ and only consider points in $E_{*}$; we denote $A=\left(p^{h}\right)^{\frac{\epsilon}{\gamma}} / p_{-\infty}^{\frac{\epsilon}{\gamma}}<1$. By assumption, we have $\epsilon \operatorname{TV}_{D}(t)<k$. Then, (5.25) implies $A \geq \frac{1}{2}$. By the elementary identity $A-1=\log A \int_{0}^{1} A^{s} d s$ we deduce

$$
\left|\frac{\left(p^{h}\right)^{\frac{\epsilon}{\gamma}}-p_{-\infty}^{\frac{\epsilon}{\gamma}}}{\epsilon}\right| \geq \frac{1}{2 \gamma \log 2} p_{-\infty}^{\frac{\epsilon}{\gamma}} \log \frac{p_{-\infty}}{p^{h}}
$$

and then, by (5.25),

$$
p^{h} \geq p_{-\infty} e^{-\frac{K}{k} \gamma \log 2} \doteq p_{*} .
$$

At last, we are concerned with the definition of $H$. First, consider a Riemann problem with initial data (2.9), giving rise to waves $\alpha, \delta$, and $\beta$. By the definitions of $\delta_{\alpha}$ and $\delta_{\beta}$, it follows that $|\delta| \leq\left|\eta_{L}-\eta_{R}\right|+\left|\delta_{\alpha}\right|+\left|\delta_{\beta}\right|$. If the solution of the Riemann problem contains rarefaction waves, the estimate is even simpler, since in that case the entropy does not change. As a consequence,

$$
\begin{aligned}
0 \leq \eta^{h}-\eta_{*} & \leq \operatorname{TV} \eta^{h}(t) \leq \operatorname{TV} \bar{\eta}+\sum\left(\left|\delta_{\alpha}\right|+\left|\delta_{\beta}\right|\right) \\
& \leq \operatorname{TV} \bar{\eta}+C\left(p_{*}, p^{*}\right) T V_{D}(t) \leq \operatorname{TV} \bar{\eta}+K C\left(p_{*}, p^{*}\right) \doteq H,
\end{aligned}
$$

by (2.5), for some constant $C\left(p_{*}, p^{*}\right)$.

Lemma 5.2 (Global Interaction Estimates) Suppose that $\mathrm{TV}(\bar{U}) \leq K_{0}$ for some positive constant $K_{0}$ and that $\epsilon \operatorname{TV}(\bar{U})$ is sufficiently small. Then, it follows that (5.16) holds for every pair of space-like curves $J$ and $J^{\prime}$ as above and

$$
F\left(J^{\prime}\right) \leq F(J) \leq F(O) .
$$

Moreover, the approximate solution $U^{h}$ is contained in the region (4.1) for some $p_{*}, p^{*}$, and $H$.

Proof We choose $K=18 K_{0}$ in Lemma 5.1; then, we have $p_{*}, p^{*}$, and $H$. The choice of $p_{*}, p^{*}$, and $H$ determines the constants $D_{0}, D, D_{1}, D_{2}$ in Lemma 4.3; in turn, the choice 
of $D_{0}, D, D_{1}, D_{2}$ fixes $D_{*}$ and $C_{H}$. Moreover, we define $G^{*}=K_{0}$. At last, we define the constants $M_{0}, M_{1}, M_{2}$ by

$$
\begin{gathered}
0<M_{0}<\min \left\{\frac{1}{2 C_{H}}, \frac{D_{*}-D_{0}}{D}, \frac{D_{1}}{D}, \frac{D}{D_{2}}, \frac{1-D_{0}}{2 D}\right\}, \\
M_{1}=\frac{9 D_{1}}{M_{0}}, \quad M_{2}=9 D+2 M_{1} D G^{*} .
\end{gathered}
$$

In turn, $M_{0}, M_{1}$, and $M_{2}$ completely define the functionals $F$ and $G$.

We justify formulas (5.28) and (5.29) by looking for constants $M_{0}, M_{1}, M_{2}$ satisfying (4.11) (or (4.15)), (4.13), (5.9), (5.14), (5.18), (5.19) (5.20), (5.22), (5.23), provided $\epsilon \operatorname{TV}(\bar{p}, \bar{u}, \bar{\eta})$ is sufficiently small. Indeed, we impose bounds on $\epsilon G(O)$, since $G(O) \leq$ $\operatorname{18TV}(\bar{p}, \bar{u}, \bar{\eta})$ by $(5.13)$ and $(5.10)$.

We start by looking for necessary conditions about $M_{0}, M_{1}, M_{2}$. In order that conditions (4.11), (5.14), (5.22) hold, we require that $D_{0}<D_{*}<1$ and choose $M_{0}$ satisfying (5.28).

Since $1-D_{0}<1$ and we may assume $D \geq C_{H}$ by possibly enlarging $D$, then (4.13) is satisfied. About $M_{1}$, by considering the extreme case $\epsilon G(O) \rightarrow 0$, conditions (5.20), (5.23) require that $M_{1}>\max \left\{8 D_{1} / M_{0}, 2 M_{0} D_{2}+3 D\right\}=8 D_{1} / M_{0}$ by (5.28). About $M_{2}$ and conditions (5.18), (5.19), we need $M_{2}>7 D+2 M_{1} D G^{*}$. Then, we choose $M_{1}$ and $M_{2}$ satisfying (5.29). Of course, other choices of these parameters are possible, leading to slightly different bounds on $\epsilon G(O)$; however, we are not aiming at optimal bounds.

We are then left to impose that $\epsilon G(O)$ is small in order that (4.11), (5.9), (5.18), (5.19) (5.20), (5.22), (5.23) are satisfied. Condition (5.20) reads

$$
\epsilon M_{3} G(O) \leq \frac{1}{2}
$$

This implies (5.9), (5.18), and (5.19). At last, we are left with (4.11), (5.22), (5.23); we conclude by requiring

$$
\epsilon G(O) \leq \min \left\{2\left(\frac{D_{*}-D_{0}}{D}-M_{0}\right), \frac{1}{2 M_{3}}, \frac{1-D_{0}-2 M_{0} D}{D+2 M_{3} D_{1}}, \frac{M_{1}-2 M_{0} D_{2}-3 D}{2 M_{3} D}\right\} .
$$

At last, the inequalities $\operatorname{TV}_{D}(J) \leq K=18 K_{0}$ and $G(O) \leq G^{*}=K_{0}$ follow by Proposition 5.1.

Lemma 5.3 Under the same assumptions of Lemma 5.2, we have

$$
3 \epsilon \sum_{\mathrm{P}} Q(\mathrm{P}) \leq F(O),
$$

where the summation is done over all the interaction points.

Proof Let $J^{\prime}$ and $J$ be space-like curves as above. By (5.16), we deduce

$$
3 \epsilon Q(\mathrm{P}) \leq F(J)-F\left(J^{\prime}\right) .
$$

By summing up over all interaction points located between $O$ and $J^{\prime}$, we have

$$
3 \epsilon \sum_{\mathrm{P} \text { between } O \text { and } J^{\prime}} Q(\mathrm{P}) \leq F(O)-F\left(J^{\prime}\right) \leq F(O) .
$$

Since $J^{\prime}$ is an arbitrary space-like curve, we have proved the lemma. 


\section{Decomposition by paths}

In this section, we introduce the notion of path in an approximate solution $U^{h}(x, t)$. Roughly speaking, a path is a sequence $\mathrm{P}\left(x_{0}, t_{0}\right), \mathrm{P}\left(x_{1}, t_{1}\right), \ldots, \mathrm{P}\left(x_{n}, t_{n}\right)$ of interaction points (apart from the case $t_{0}=0$ ) in the $x t$-plane, with $0 \leq t_{0}<t_{1}<\cdots<t_{n}$, which are connected by shock or entropy waves. By writing $\mathrm{P}_{j}=\mathrm{P}\left(x_{j}, t_{j}\right)$, a path is represented by

$$
\Gamma: \mathrm{P}_{0} \rightarrow \mathrm{P}_{1} \rightarrow \cdots \rightarrow \mathrm{P}_{n}
$$

and the segment $\mathrm{P}_{j-1} \mathrm{P}_{j}$ is called a front. If a shock or an entropy front starts at $\mathrm{P}_{n-1}$ and propagates without interacting with other waves, we continue the path as a half-line following that front and denote $\mathrm{P}_{n}=\mathrm{P}_{\infty}=\infty$.

The index $\left(c_{j}, k_{j}\right)$ of the front $\mathrm{P}_{j-1} \mathrm{P}_{j}$ was defined by Asakura [3], Temple and Young [21]. We recall such definition below; the numbers $c_{j}$ and $k_{j}$ are called the type and the generation order of the front. First, a suitable positive integer, to be defined later on, is assigned to $k_{1}$. Then,

$$
\begin{aligned}
& c_{j}=\left\{\begin{array}{l}
1 \text { if } \mathrm{P}_{j-1} \mathrm{P}_{j} \text { is a 1-shock wave, } \\
2 \text { if } \mathrm{P}_{j-1} \mathrm{P}_{j} \text { is a 2-shock wave, } \\
0 \text { if } \mathrm{P}_{j-1} \mathrm{P}_{j} \text { is an entropy wave, }
\end{array} \text { for } j \geq 1,\right.
\end{aligned}
$$

The sequence $\left(c_{1}, k_{1}\right),\left(c_{2}, k_{2}\right), \ldots,\left(c_{n}, k_{n}\right)$ is called the index of the path. If $\mathrm{P}_{0} \in\{t=0\}$, then we set $k_{1}=1$ and the path is called a primary path; it is denoted by $\Gamma^{P}: \mathrm{P}_{0} \rightarrow \mathrm{P}_{1} \rightarrow$ $\cdots \rightarrow \mathrm{P}_{n}$ and each $\mathrm{P}_{j-1} \mathrm{P}_{j}$ is a primary front.

Shock and entropy waves interact with other waves and generate new waves as described in Lemma 4.3. If an interaction $\theta^{\prime}+\theta^{\prime \prime}$ occurs at some point $\mathrm{P}_{0}$ and generates a shock or an entropy wave of amplitude $O(1) \epsilon \theta^{\prime} \theta^{\prime \prime}$, the front of that wave is called a secondary front and a secondary path starts at $\mathrm{P}_{0}$; it is denoted by $\Gamma^{S}: \mathrm{P}_{0} \rightarrow \mathrm{P}_{1} \rightarrow \cdots \rightarrow \mathrm{P}_{n}$. In this case, the initial generation order $k_{1}$ is at least 2. Secondary paths also arise when the strength of an outgoing wave is larger than that of the ingoing wave of the same family; in that case, the secondary path accounts for the difference of the strengths. In any case, secondary paths are generated only in Cases (1)-(5). The outgoing entropy wave appearing in Case (3) always gives rise to a primary path, see Remark 4.2.

The construction of the paths along with the definition of their strengths is done iteratively as follows. Below, we denote waves $\alpha, \beta, \ldots$ with front $\mathrm{P}_{j-1} \mathrm{P}_{j}$ by $\alpha: \mathrm{P}_{j-1} \mathrm{P}_{j}, \beta: \mathrm{P}_{j-1} \mathrm{P}_{j}$ and so on. We assume for the moment that all waves involved in the interactions under consideration are physical, see Sect. 3; non-physical waves are considered at the end of the construction.

First, we focus on the time interval ranging from $t=0$ to the first interaction time $t_{1}$. Consider the case of a shock or entropy wave $\theta$ issuing from $\mathrm{P}_{0} \in\{t=0\}$ and interacting with another wave at $\mathrm{P}_{1}$. The front $\mathrm{P}_{0} \mathrm{P}_{1}$ forms a primary path $\Gamma^{P}: \mathrm{P}_{0} \rightarrow \mathrm{P}_{1}$. The strength of $\Gamma^{P}$ is $\alpha$ if $\theta=\alpha$ (or $\beta$ if $\theta=\beta$ ) and $M_{0}|\delta|$ if $\theta=\delta$; the generation order is 1 .

Next, $t=t_{1}$. Suppose first that a shock wave $\alpha: \mathrm{P}_{0} \mathrm{P}_{1}$ interacts with another wave at $\mathrm{P}_{1}$ and generates $\theta_{1}^{\prime}: \mathrm{P}_{1} \mathrm{P}_{2}, \theta_{2}^{\prime}: \mathrm{P}_{1} \mathrm{P}_{2}^{\prime}$ and $\delta^{\prime}: \mathrm{P}_{1} \mathrm{P}_{2}^{\prime \prime}$. We only consider Cases (1), (3), (4), and (6) in Lemma 4.3. Below, we denote some generic constants that may change from line to line by

$$
D^{\prime}, D^{\prime \prime} \in[0, D], \quad D_{1}^{\prime} \in\left[0, D_{1}\right], \quad D_{2}^{\prime \prime} \in\left[0, D_{2}\right], \quad D_{*}^{\prime} \in\left[0, D_{*}\right]
$$


(1): $\beta+\alpha \rightarrow \alpha^{\prime}+\delta^{\prime}+\beta^{\prime}$. If $\alpha^{\prime} \leq \alpha$, we decompose $\Gamma^{P}$ into the paths $\Gamma_{1}^{P}$ and $\Gamma_{2}^{P}$, which have the same index of $\Gamma^{P}$ but strengths $\alpha^{\prime}$ and $\alpha-\alpha^{\prime}$, respectively. The path $\Gamma_{1}^{P}$ is extended to $\mathrm{P}_{2}$ with unchanged generation order and strength; the path $\Gamma_{2}^{P}$ stops at $\mathrm{P}_{1}$. If $\alpha^{\prime}>\alpha$, we have $\alpha^{\prime}-\alpha=\epsilon D^{\prime} \alpha \beta$; we extend $\Gamma^{P}$ in the same direction with strength $\alpha$ and generate a secondary path $\Gamma_{1}^{S}: \mathrm{P}_{1} \rightarrow \mathrm{P}_{2}$ with index $(1,2)$ and strength $\epsilon D^{\prime} \alpha \beta$. The paths related to $\beta$ are dealt analogously.

An entropy wave $\delta^{\prime}$ with strength $M_{0}\left|\delta^{\prime}\right|=\epsilon D_{2}^{\prime \prime} \alpha \beta$ is generated; we define a secondary path $\Gamma_{0}^{S}: \mathrm{P}_{1} \rightarrow \mathrm{P}_{2}^{\prime \prime}$ with index $(0,2)$ and strength $\epsilon M_{0} D_{2}^{\prime \prime} \alpha \beta$.

(3): $\alpha_{1}+\alpha_{2} \rightarrow \alpha^{\prime}+\delta^{\prime}+\pi^{\prime}$. Assume for instance that $\alpha=\alpha_{1}$. We have $\alpha^{\prime}-\left(\alpha_{1}+\alpha_{2}\right)=$ $\epsilon D^{\prime} \alpha_{1} \alpha_{2}$; then, we extend $\Gamma^{P}$ in the same direction with strength $\alpha_{1}$ and generate a secondary path $\Gamma_{1}^{S}: \mathrm{P}_{1} \rightarrow \mathrm{P}_{2}$ with index $(1,2)$ and strength $\epsilon D^{\prime} \alpha_{1} \alpha_{2}$.

An entropy wave $\delta^{\prime}$ with strength $\left|\delta^{\prime}\right|=D_{2}^{\prime \prime} \alpha_{1} \alpha_{2}$ is generated. We define a primary path $\Gamma_{0}^{P}: \mathrm{P}_{1} \rightarrow \mathrm{P}_{2}^{\prime \prime}$ with index $(0,2)$ and strength $M_{0} D_{2}^{\prime \prime} \alpha_{1} \alpha_{2}$.

(4): $\delta+\alpha \rightarrow \alpha^{\prime}+\delta^{\prime}+\theta^{\prime}$. If $\alpha^{\prime} \leq \alpha$, we proceed exactly as in the first part of Case (1). If $\alpha^{\prime}>\alpha$, we have $\alpha^{\prime}-\alpha=\epsilon D_{1}^{\prime} \alpha|\delta|$. Then, we extend $\Gamma^{P}$ in the same direction with strength $\alpha$ and generate a secondary path $\Gamma_{1}^{S}: \mathrm{P}_{1} \rightarrow \mathrm{P}_{2}$ with index $(1,2)$ and strength $\epsilon D_{1}^{\prime} \alpha|\delta|$.

If $\theta^{\prime}=\beta^{\prime}$, then $\beta^{\prime}=\epsilon D_{1}^{\prime} \alpha|\delta|$ and we have another secondary path $\Gamma_{2}^{S}: \mathrm{P}_{1} \rightarrow \mathrm{P}_{2}^{\prime}$ with index $(2,2)$ and strength $\epsilon D_{1}^{\prime} \alpha|\delta|$. The entropy wave $\delta$ constitutes a primary path $\Gamma_{0}^{P}: \mathrm{P}_{0}^{\prime} \rightarrow \mathrm{P}_{1}$. If $\left|\delta^{\prime}\right| \leq|\delta|$, we decompose $\Gamma_{0}^{P}$ into the paths $\Gamma_{0,1}^{P}$ and $\Gamma_{0,2}^{P}$, which have the same index of $\Gamma_{0}^{P}$ but strengths $M_{0}\left|\delta^{\prime}\right|$ and $M_{0}\left(\left|\delta^{\prime}\right|-|\delta|\right)$, respectively. The path $\Gamma_{0,1}^{P}$ is extended to $\mathrm{P}_{2}^{\prime \prime}$ with unchanged generation order and strength, and the path $\Gamma_{0,2}^{P}$ is stopped. If $\left|\delta^{\prime}\right|>|\delta|$ and then $\left|\delta^{\prime}\right|-|\delta|=\epsilon D^{\prime \prime} \alpha|\delta|$, we have a secondary path $\Gamma_{0}^{S}: \mathrm{P}_{1} \rightarrow \mathrm{P}_{2}^{\prime \prime}$ with index $(0,2)$ and strength $\epsilon M_{0} D^{\prime \prime} \alpha|\delta|$.

(6): $\xi+\alpha \rightarrow \alpha^{\prime}+\delta^{\prime}+\beta^{\prime}$. By Lemma 4.3 and (4.12), we find $\zeta \geq \xi$ and $D_{*}^{\prime} \leq D_{*}$ such that

$$
\alpha^{\prime}=\alpha-\zeta, \quad \beta^{\prime}+M_{0}\left|\delta^{\prime}\right|=D_{*}^{\prime} \zeta .
$$

We define $\zeta_{2}, \zeta_{0}$ by $D_{*}^{\prime} \zeta_{2}=\beta^{\prime}, D_{*}^{\prime} \zeta_{0}=M_{0}\left|\delta^{\prime}\right|$ so that

$$
\zeta=\zeta_{2}+\zeta_{0}, \quad \zeta_{2}: \zeta_{0}=\beta^{\prime}: M_{0}\left|\delta^{\prime}\right| \text {. }
$$

The path $\Gamma^{P}$ is decomposed into three paths $\Gamma_{1}^{P}, \Gamma_{2}^{P}$, and $\Gamma_{0}^{P}$, whose strengths are $\alpha-\zeta, \zeta_{2}$, and $\zeta_{0}$, respectively. The path $\Gamma_{1}^{P}$ is extended to $\mathrm{P}_{2}$ with index $(1,1)$ and strength $\alpha-\zeta$. The path $\Gamma_{2}^{P}$ is extended to $\mathrm{P}_{2}^{\prime}$ with index $(2,2)$ and strength $\beta^{\prime}=D_{*}^{\prime} \zeta_{2}$. Finally, $\Gamma_{0}^{P}$ is extended to $\mathrm{P}_{0} \rightarrow \mathrm{P}_{1} \rightarrow \mathrm{P}_{2}^{\prime \prime}$, where $\mathrm{P}_{1} \mathrm{P}_{2}^{\prime \prime}$ has index $(0,2)$ and strength $M_{0}\left|\delta^{\prime}\right|=D_{*}^{\prime} \zeta_{0}$. No secondary path shows up in this case.

Remark 6.1 In Cases (1) and (4), both possibilities $\alpha^{\prime} \leq \alpha$ and $\alpha^{\prime}>\alpha$ may occur [4].

Now, suppose that an entropy wave $\delta: \mathrm{P}_{0} \mathrm{P}_{1}$ interacts with another wave at $\mathrm{P}_{1}$; in this case, we already have a primary path $\Gamma_{0}^{P}: \mathrm{P}_{0} \rightarrow \mathrm{P}_{1}$. The paths due to the interaction of $\delta$ with a 1 -shock wave have been already defined in Case (4). In the case $\delta$ interacts with a 1-rarefaction wave, the definitions are analogous (see Lemma 4.3, Case (5)).

Then, $t=t_{n}$. We suppose that the paths have been constructed up to the interaction time $t=t_{n}$. First, we define the generation order of a shock and entropy wave $\gamma$. The front of $\gamma$ belongs to a finite number $N$ of paths, which can be ordered by their increasing generation order as follows:

$$
\Gamma_{1}^{P}, \ldots \Gamma_{p}^{P}, \Gamma_{p+1}^{S}, \ldots \Gamma_{N}^{S}, \text { for } k_{1}^{P} \leq \cdots \leq k_{p}^{P}, k_{p+1}^{S} \leq \cdots \leq k_{N}^{S} .
$$


Here, $k_{m}^{P}, k_{m}^{S}$ denote the generation orders of the front; we drop the indexes $P$ and $S$ in the following. The generation order of $\gamma$ is defined by

$$
k_{\gamma}=\min _{1 \leq l \leq N}\left\{k_{l}\right\} .
$$

We now accomplish the construction of the decomposition by paths. Consider a path $\Gamma: \mathrm{P}_{0} \rightarrow \mathrm{P}_{1} \rightarrow \cdots \rightarrow \mathrm{P}_{n}$ to $t=t_{n}$; we first assume that $\mathrm{P}_{n-1}$ and $\mathrm{P}_{n}$ are connected by a shock $\alpha$. The front $\mathrm{P}_{n-1} \mathrm{P}_{n}$ belongs to some paths $\Gamma_{l}$, for $1 \leq l \leq N$; the path $\Gamma_{l}$ has index $\left(1, k_{l}\right)$ and strength $\alpha_{l}$. Of course, the path $\Gamma$ is one of the $\Gamma_{l}$ 's. By (6.3), the strength $\alpha$ is decomposed into

$$
\alpha=\sum_{l=1}^{N} \alpha_{l}
$$

Denote by $\alpha^{\prime}: \mathrm{P}_{n} \mathrm{P}_{n+1}, \beta^{\prime}: \mathrm{P}_{n} \mathrm{P}_{n+1}^{\prime}, \delta^{\prime}: \mathrm{P}_{n} \mathrm{P}_{n+1}^{\prime \prime}$ the waves produced by the interaction of $\alpha$ with another wave at $\mathrm{P}_{n}$. Let $k_{*}$ denote either $\max \left\{k_{\alpha}, k_{\beta}\right\}$ or $\max \left\{k_{\alpha}, k_{\delta}\right\}$. Below, we omit for brevity Cases (3) and (4), since they are analogous to Case (1); see [5] for complete details on the algorithm.

(1): $\beta+\alpha \rightarrow \alpha^{\prime}+\delta^{\prime}+\beta^{\prime}$. If $\alpha^{\prime} \leq \alpha$, there exists $1 \leq r \leq N$ and $0 \leq \bar{\alpha}_{r}<\alpha_{r}$ such that $\alpha-\alpha^{\prime}=\bar{\alpha}_{r}+\sum_{l=r+1}^{N} \alpha_{l}$. We split $\Gamma_{r}$ into the paths $\Gamma_{r}^{(1)}$ and $\Gamma_{r}^{(2)}$, so that the orders of the paths are unchanged while their strengths $\alpha_{r}^{(1)}$ and $\alpha_{r}^{(2)}$ are decomposed according to the proportion

$$
\alpha_{r}^{(2)}: \alpha_{r}^{(1)}=\bar{\alpha}_{r}:\left(\alpha_{r}-\bar{\alpha}_{r}\right)
$$

Then, we extend $\Gamma_{l}, 1 \leq l \leq r-1$, and $\Gamma_{r}^{(1)}$ to $\mathrm{P}_{n+1}$ with orders and strengths unchanged. The paths $\Gamma_{r}^{(\overline{2})}$ and $\Gamma_{l}, r+1 \leq l \leq N$, stop.

If $\alpha^{\prime}>\alpha$, we have $\alpha^{\prime}-\alpha=\epsilon D^{\prime} \alpha \beta$; we extend each $\Gamma_{l}$ in the same direction with the same strength and generate a secondary path $\Gamma_{1}^{S}: \mathrm{P}_{n} \rightarrow \mathrm{P}_{n+1}$ with index $\left(1, k_{*}+1\right)$ and strength $\epsilon D^{\prime} \alpha \beta$.

If $\beta^{\prime}>\beta$, we have $\beta^{\prime}-\beta=\epsilon D^{\prime} \alpha \beta$; we generate a secondary path $\Gamma_{2}^{S}: \mathrm{P}_{n} \rightarrow$ $\mathrm{P}_{n+1}^{\prime}$ with index $\left(2, k_{*}+1\right)$ and strength $\epsilon D^{\prime} \alpha \beta$. At last, an entropy wave of strength $\left|\delta^{\prime}\right|=\epsilon D_{2}^{\prime \prime} \alpha \beta$ is generated and we have a secondary path $\Gamma_{0}^{S}: \mathrm{P}_{n} \rightarrow \mathrm{P}_{n+1}^{\prime \prime}$ with index $\left(0, k_{*}+1\right)$ and strength $\epsilon M_{0} D_{2}^{\prime \prime} \alpha \beta$.

(6): $\xi+\alpha \rightarrow \alpha^{\prime}+\delta^{\prime}+\beta^{\prime}$. No secondary path is generated in this case. As in the first step, there are positive quantities $\zeta$ and $D_{*}^{\prime}$ satisfying (6.1); we also fix $\zeta_{2}$ and $\zeta_{0}$ satisfying (6.2). Then, there are $1 \leq N_{1} \leq N_{2} \leq N$ and $\bar{\alpha}_{N_{1}}, \bar{\alpha}_{N_{2}}$ with $0 \leq \bar{\alpha}_{N_{1}}<\alpha_{N_{1}}, 0 \leq \bar{\alpha}_{N_{2}}<\alpha_{N_{2}}$ such that

$$
\alpha^{\prime}=\sum_{l=1}^{N_{1}} \alpha_{l}-\bar{\alpha}_{N_{1}}, \quad \zeta_{2}=\bar{\alpha}_{N_{1}}+\sum_{l=N_{1}+1}^{N_{2}} \alpha_{l}-\bar{\alpha}_{N_{2}}, \quad \zeta_{0}=\bar{\alpha}_{N_{2}}+\sum_{l=N_{2}+1}^{N} \alpha_{l} .
$$

(a) We extend every $\Gamma_{l}$ with $1 \leq l \leq N_{1}-1$ to $\mathrm{P}_{n+1}$ with indices and strengths unchanged. Primary paths remain primary and so secondary.

Then, we split $\Gamma_{N_{1}}$ into $\Gamma_{N_{1}}^{(1)}$ and $\Gamma_{N_{1}}^{(2)}$, so that

$$
\alpha_{N_{1}}^{(1)}+\alpha_{N_{1}}^{(2)}=\alpha_{N_{1}}, \quad \alpha_{N_{1}}^{(1)}: \alpha_{N_{1}}^{(2)}=\left(\alpha_{N_{1}}-\bar{\alpha}_{N_{1}}\right): \bar{\alpha}_{N_{1}} .
$$


Therefore, $\alpha_{N_{1}}^{(1)}=\alpha_{N_{1}}-\bar{\alpha}_{N_{1}}$ and $\alpha_{N_{1}}^{(2)}=\bar{\alpha}_{N_{1}}$. Let $k_{N_{1}}$ be the order of $\Gamma_{N_{1}}$ in $\mathrm{P}_{n-1} \mathrm{P}_{n}$. We extend $\Gamma_{N_{1}}^{(1)}$ to $\mathrm{P}_{n+1}$ with index $\left(2, k_{N_{1}}\right)$ and strength $\alpha_{N_{1}}^{(1)}$. Then, we extend $\Gamma_{N_{1}}^{(2)}$ to $\mathrm{P}_{n+1}^{\prime}$ with index $\left(2, k_{N_{1}}+1\right)$ and strength $D_{*}^{\prime} \alpha_{N_{1}}^{(2)}=D_{*}^{\prime} \bar{\alpha}_{N_{1}}$.

(b) Similarly, $\Gamma_{N_{2}}$ is split into $\Gamma_{N_{2}}^{(1)}$ and $\Gamma_{N_{2}}^{(2)}$ so that

$$
\alpha_{N_{2}}^{(1)}+\alpha_{N_{2}}^{(2)}=\alpha_{N_{2}}, \quad \alpha_{N_{2}}^{(1)}: \alpha_{N_{2}}^{(2)}=\left(\alpha_{N_{2}}-\bar{\alpha}_{N_{2}}\right): \bar{\alpha}_{N_{2}} .
$$

We extend the paths $\Gamma_{l}$ for $N_{1}+1 \leq l \leq N_{2}-1$ to $\mathrm{P}_{n+1}^{\prime}$ with index $\left(2, k_{l}+1\right)$ and strength $D_{*}^{\prime} \alpha_{l}$. We also extend $\Gamma_{N_{2}}^{(1)}$ to $\mathrm{P}_{n+1}$ with index $\left(2, k_{N_{2}}+1\right)$ and strength $D_{*}^{\prime} \alpha_{N_{2}}^{(1)}$ as well as $\Gamma_{N_{2}}^{(2)}$ to $\mathrm{P}_{n+1}^{\prime \prime}$ with index $\left(0, k_{N_{2}}+1\right)$ and strength $D_{*}^{\prime} \alpha_{N_{2}}^{(2)}$ (entropy wave).

(c) At last, we extend the paths $\Gamma_{l}$ for $l \geq N_{2}+1$ to $\mathrm{P}_{n+1}^{\prime \prime}$ with index $\left(0, k_{l}+1\right)$ and strength $D_{*}^{\prime} \alpha_{l}$.

Now, we assume that $\mathrm{P}_{n-1}$ and $\mathrm{P}_{n}$ are connected by an entropy wave $\delta$, which interacts with another wave at $\mathrm{P}_{n}$ and generates $\alpha^{\prime}: \mathrm{P}_{n} \mathrm{P}_{n+1}, \beta^{\prime}: \mathrm{P}_{n} \mathrm{P}_{n+1}^{\prime}$ and $\delta^{\prime}: \mathrm{P}_{n} \mathrm{P}_{n+1}^{\prime \prime}$. In this case, there is a path $\Gamma_{0}: \mathrm{P}_{n-1} \rightarrow \mathrm{P}_{n}$. If $\delta$ interacts with a 1 -shock wave, we refer to the Case (4) above. If $\delta: \mathrm{P}_{n-1} \mathrm{P}_{n}$ interacts with a 1-rarefaction wave, we define the paths in a completely analogous way. Therefore, we have completed the construction of paths involving only physical waves.

At last, we discuss the case of non-physical waves. The generation order of a non-physical wave is defined as in [7]. About paths, if a shock wave $\alpha$ or an entropy wave $\delta$ belongs to a path $\Gamma_{l}$ and the interaction generates a non-physical wave, then the path $\Gamma_{l}$ is simply extended in the previous direction with the same index and strength.

A collection of paths $\Gamma=\left\{\Gamma_{l}\right\}$, which are divided into primary paths $\Gamma^{\boldsymbol{P}}=\left\{\Gamma_{l}^{P}\right\}$ and secondary paths $\Gamma^{S}=\left\{\Gamma_{l}^{S}\right\}$, is then defined up to the next interaction time $t_{n+1}$ and hence as long as the approximate solution exists. This concludes the definition of the paths. Remark that the construction above implicitly defines the initial generation order $k_{1}$ of a path, a quantity that was not previously fixed.

We now introduce analogous definitions for rarefaction waves, which however are not related to paths.

We assign a generation order to the approximate rarefaction waves as follows; our definition slightly differs from that given in [7]. Recall that in the approximate solutions, a rarefaction wave of size $\theta$ is split into $N=[\theta / h]+1$ fronts, each of them having strength $\theta / N<h$, [7]. We assign order 1 to any of these rarefaction fronts issuing from $t=0$.

Suppose that a rarefaction front $\xi$ with generation order $k$ interacts with a wave $\theta$ with generation order $k^{\prime}$ and the waves $\theta_{1}^{\prime}, \delta^{\prime}, \theta_{2}^{\prime}$ are produced. If $\theta_{1}^{\prime}=\xi^{\prime}$, the generation order of $\xi^{\prime}$ is defined to be $k$. If $\theta_{2}^{\prime}=\pi^{\prime}$ and $\theta \neq \pi$, its generation order is $\max \left\{k, k^{\prime}\right\}+1$. Lemma 4.3 states that the interaction of two shock waves of the same family, Case (3), and possibly that of a shock wave and an entropy wave, Case (4), also generates a rarefaction wave. In these cases, by denoting $k, k^{\prime}$ the generation orders of $\alpha_{1}, \alpha_{2}$ or $\delta, \alpha$, respectively, the generation order of $\pi^{\prime}$ is defined to be $\max \left\{k, k^{\prime}\right\}+1$.

When a rarefaction wave $\xi$ of generation order $k$ interacts with another wave and produces $\xi^{\prime}$, the amplitude of $\xi^{\prime}$ can exceed $h$. In this case, we have to divide $\xi^{\prime}$ into $\xi_{1}^{\prime}+\xi_{2}^{\prime}$ so that $\xi_{j} \leq h, j=1,2$. That is the reason why we do not construct paths for rarefaction waves. We will assign the same generation order $k$ to both $\xi_{j}^{\prime}, j=1,2$.

We call secondary rarefaction waves those rarefactions that are generated in Cases (4), (5) and (10). Secondary rarefaction waves were called reflected in [5] because of the special 


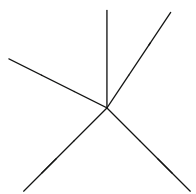

(1)

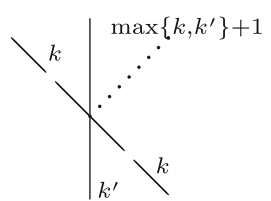

(5)

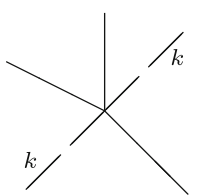

(2)

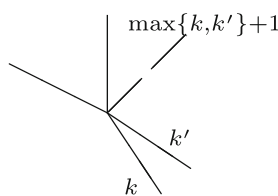

(3)

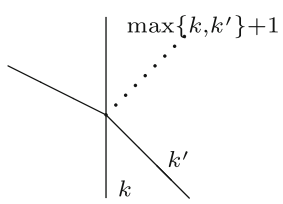

(4)

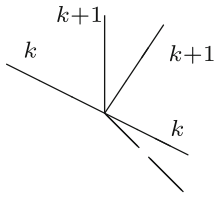

(6)

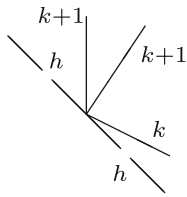

(7)

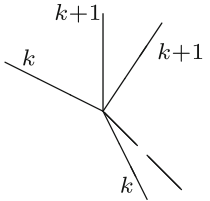

(8)

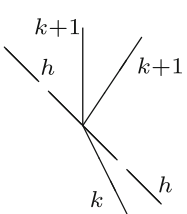

(9)

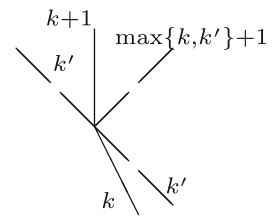

(10)

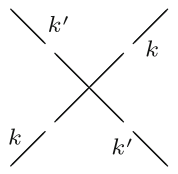

(11)

Fig. 3 Generation orders for the cases in Lemma 4.3. Solid lines indicate shock or entropy waves; broken lines represent rarefaction waves; dotted lines denote either shock or rarefaction waves

interaction patterns; we do not use this terminology here because of the reflected rarefaction waves of Case (3), which need a particular treatment.

By the above construction and definitions, we deduce the following proposition, where we use the definition (5.15) of $Q(\mathrm{P})$ (Fig. 3).

Proposition 6.1 The amount of secondary rarefaction waves or secondary paths that are generated at an interaction point $\mathrm{P}$ is estimated, for each family, by $\in Q(P)$.

\section{Estimates along paths}

In this section, we prove several results about the decay of waves along paths. As we saw in the previous section, paths only involve shock or entropy waves; we denote either of these waves by $\sigma$. By the estimates along paths, we provide a bound on the total amount of rarefaction waves.

Let $\Gamma: \mathrm{P}_{0} \rightarrow \mathrm{P}_{1} \rightarrow \cdots \rightarrow \mathrm{P}_{n}$ be a path, with $\mathrm{P}_{i}$ occurring at time $t_{i}$. We denote by $\mathrm{P}_{\Gamma}$ $\left(t_{0, \Gamma}\right)$ the point (respectively, the time) where the path $\Gamma$ is generated. We denote by $c_{m}, k_{m}$, and $\sigma_{m}$ the type, generation order, and strength of the front $\mathrm{P}_{m-1} \mathrm{P}_{m}$ in $\Gamma$, respectively; the sequences $c_{\Gamma}=\left\{c_{m}\right\}, k_{\Gamma}=\left\{k_{m}\right\}$, and $\sigma_{\Gamma}=\left\{\sigma_{m}\right\}$ are the type, generation order and strength of $\Gamma$. We consider the path $\Gamma$ as a Lipschitz curve $x=\Gamma(t)$; then, the type, order, and strength of $\Gamma$ are piecewise constant functions of $t$, denoted by $c_{\Gamma}(t), k_{\Gamma}(t), \sigma_{\Gamma}(t)$, respectively. Here, we set $\sigma_{\Gamma}\left(t_{i}\right)=\sigma_{\Gamma}\left(t_{i}-\right)$ for $i=1,2, \ldots, n, \sigma_{\Gamma}\left(t_{0, \Gamma}\right)=\sigma_{\Gamma}\left(t_{1}\right)$ and so on for $c_{\Gamma}$ and $k_{\Gamma}$. We also denote with a slight abuse of notation, for $i=1, \ldots, n$,

$$
\sigma_{\Gamma}\left(\mathrm{P}_{i}\right)=\sigma_{\Gamma}\left(t_{i}-\right)
$$


We denote by $\boldsymbol{\Gamma}$ the collection of all paths. Moreover, for every shock or entropy wave $\sigma$, we denote by $\boldsymbol{\Gamma}_{\sigma}$ the collection of paths, which the front of $\sigma$ belongs to. We now state a result about the decomposition of waves, whose proof is obvious by the construction of paths.

Lemma 7.1 Consider any approximate solution, a time $t$ where no interaction occurs, a shock or an entropy wave $\sigma$ at time $t$. Then,

$$
\sum_{\Gamma \in \boldsymbol{\Gamma}_{\sigma}} \sigma_{\Gamma}(t)=\left\{\begin{array}{ll}
\sigma & \text { if } \sigma \text { is a shock, } \\
M_{0}|\sigma| & \text { if } \sigma \text { is an entropy wave. }
\end{array} .\right.
$$

For typographical reasons, we denote

$$
c=D_{*} .
$$

Lemma 7.2 Consider any approximate solution and any path $\Gamma: \mathrm{P}_{0} \rightarrow \mathrm{P}_{1} \rightarrow \cdots \rightarrow \mathrm{P}_{n}$. Let $k_{m}$ be the generation order and $\sigma_{m}$ the strength of the front $\mathrm{P}_{m-1} \mathrm{P}_{m}$ in $\Gamma$. Then,

$$
\begin{aligned}
& k_{m+1}=k_{m} \quad \Rightarrow \sigma_{m+1}=\sigma_{m}, \\
& k_{m+1}=k_{m}+1 \Rightarrow \sigma_{m+1} \leq c \sigma_{m} .
\end{aligned}
$$

Proof The paths are constructed in such a way to satisfy the condition above. In particular, formula (6.1) shows that $\sigma_{m+1}$ does not exceed $c \sigma_{m}$.

We now introduce the total amount of shock (and entropy) waves at time $t$ :

$$
\begin{aligned}
L^{-}(t) & =\sum\{\alpha(t)+\beta(t)\}, \\
L(t) & =\sum\left\{\alpha(t)+\beta(t)+M_{0}|\delta(t)|\right\},
\end{aligned}
$$

where the sum is performed over all shock (and entropy, resp.) waves occurring in the approximate solution at time $t$. Lemma 7.2 clearly implies the following result.

Lemma 7.3 (Pathwise version of Nishida's lemma, [16]) For any approximate solution and $t$ different from interaction times, we have

1. $\sigma_{\Gamma}(t) \leq c^{k_{\Gamma}(t)-k_{\Gamma}\left(t^{\prime}\right)} \sigma_{\Gamma}\left(t^{\prime}\right)$ for any $\Gamma \in \Gamma$ and $0 \leq t^{\prime} \leq t$,

2. $L(t)=\sum_{\Gamma \in \Gamma} \sigma_{\Gamma}(t)$.

For any $\Gamma \in \Gamma$, we denote by $t_{0, \Gamma}, P_{0, \Gamma}$ the time, resp. the point, at which $\Gamma$ is generated. Then, by the above lemma

$$
\sigma_{\Gamma}(t) \leq c^{k_{\Gamma}(t)-k_{\Gamma}\left(t_{0, \Gamma}\right)} \sigma_{\Gamma}\left(t_{0, \Gamma}\right) .
$$

Let $t$ be different from interaction times. For $k=1,2, \ldots$, we denote the total amount of the strengths at time $t$ of all primary paths whose generation order at time $t$ is $k$ (resp., $k$ and more) by

$$
L_{k}^{P}(t)=\sum_{\Gamma \in \Gamma^{P}, k_{\Gamma}(t)=k} \sigma_{\Gamma}(t), \quad V_{k}^{P}(t)=\sum_{l \geq k} L_{l}^{P}(t) .
$$

We also define for $k \geq 2$

$V_{k}^{S}(t)$ : the total amount at time $t$ of the strengths of both secondary paths and secondary rarefaction waves generated at times $t^{\prime} \leq t$, whose generation orders are larger than or equal to $k$. 
We denote $F(0)=F(O)$ and observe that by Lemma 5.3 we have

$$
V_{k}^{S}(t) \leq F(0)
$$

for every $k \geq 2$ and every $t$.

Proposition 7.1 For every approximate solution and $k \geq 1$, we have

$$
\begin{aligned}
L_{k}^{P}(t) & \leq c^{k-1} L(0), \\
V_{k}^{P}(t) & \leq \frac{c^{k-1}}{1-c} L(0) .
\end{aligned}
$$

Proof If $\Gamma \in \Gamma^{P}$, then $t_{0, \Gamma}=0$. Since $k_{\Gamma}(0)=1$, we deduce $\sigma_{\Gamma}(t) \leq c^{k_{\Gamma}(t)-1} \sigma_{\Gamma}(0)$ by (7.5). Then, for any $k \geq 1$,

$$
\sum_{\substack{\Gamma \in \Gamma^{P} \\ k_{\Gamma}(t)=k}} \sigma_{\Gamma}(t) \leq c^{k-1} \sum_{\Gamma \in \Gamma^{P}} \sigma_{\Gamma}(0)=c^{k-1} L(0) .
$$

Formula (7.8) follows by $\sum_{l \geq k} c^{l-1}=\frac{c^{k-1}}{1-c}$.

Let us denote

$$
\mathcal{F}(0)=L(0)+F(0) .
$$

Proposition 7.2 For every approximate solution, we have

$$
L^{-}(t) \leq \mathcal{F}(0) \text {. }
$$

Proof The amount of primary fronts for shock waves at $t=0$ equals $L^{-}(0) \leq L(0)$. If a secondary front $\Gamma^{S}$ of a shock wave is generated at point $\mathrm{P}$, then the strength of $\Gamma^{S}$ in that segment is less than $\epsilon Q(\mathrm{P})$. As a consequence, the result follows by Lemmas 7.1 and 5.3.

We now prove a lemma that is stronger than Lemma 5.3 because the estimate is independent of $\epsilon$. This result will be used to prove Proposition 8.1, which shows that the approximate solution is defined for all $0 \leq t<\infty$.

Lemma 7.4 Under the same assumptions of Lemma 5.2, we have

$$
\sum_{\mathrm{P}} Q(\mathrm{P}) \leq \frac{1}{6} Q(0)+\frac{c M_{3}}{6(1-c)} G^{*} \mathcal{F}(0),
$$

where the summation is done over all the interaction points.

Proof Let $J^{\prime}$ and $J$ be space-like curves and assume that there is a single interaction point $\mathrm{P}$ between $J^{\prime}$ and $J$.

In Cases (1), (3), and (4), by virtue of (5.18), (5.19) and (5.20), we deduce

$$
6 Q(\mathrm{P}) \leq Q(J)-Q\left(J^{\prime}\right) .
$$

In Case (6), we proved in (5.21) that

$$
M_{1} \alpha \xi \leq Q(J)-Q\left(J^{\prime}\right)+2 M_{3}\left(\beta^{\prime}+M_{0}\left|\delta^{\prime}\right|\right) G(J),
$$

where $\beta^{\prime}, \delta^{\prime}$ are outgoing waves generated by the interaction at $\mathrm{P}$ and denoted from now on by $\beta^{\prime}(\mathrm{P}), \delta^{\prime}(\mathrm{P})$, respectively. By (5.28), (5.29) and Lemma 5.2, we obtain

$$
9 Q(\mathrm{P}) \leq Q(J)-Q\left(J^{\prime}\right)+2 M_{3}\left\{\beta^{\prime}(\mathrm{P})+M_{0}\left|\delta^{\prime}(\mathrm{P})\right|\right\} G(O) .
$$


About Case (10), the outgoing 2-wave generated by the interaction is a rarefaction wave $\pi^{\prime}$; in the following, we denote both a shock and a rarefaction wave by $\theta^{\prime}$. Summing up over all interaction points $\mathrm{P}$ between $O$ and $J^{\prime}$, by (7.11) and (7.12), we deduce

$$
\begin{aligned}
\sum_{\mathrm{P} \text { between } O \text { and } J^{\prime}} 6 Q(\mathrm{P}) \leq & Q(O)-Q\left(J^{\prime}\right) \\
& +2 M_{3} G(O) \sum_{\substack{\text { P between } O \text { and } J^{\prime} \\
\text { Cases (6)-(10) }}}\left\{\theta^{\prime}(\mathrm{P})+M_{0}\left|\delta^{\prime}(\mathrm{P})\right|\right\} .
\end{aligned}
$$

Next, we claim that

$$
\sum_{\substack{\text { P between } O \text { and } J^{\prime} \\ \text { Cases (6) -(10) }}}\left\{\theta^{\prime}(\mathrm{P})+M_{0}\left|\delta^{\prime}(\mathrm{P})\right|\right\} \leq \frac{c}{1-c} \mathcal{F}(0) .
$$

Indeed, in Cases (6)-(10), we have $\theta^{\prime}+M_{0}\left|\delta^{\prime}\right| \leq c \alpha$ by (4.14) and (7.3). Then, by (7.5), we have

$$
\begin{aligned}
\sum_{\substack{\mathrm{P} \text { between } O \text { and } J^{\prime} \\
\text { Cases (6) - (10) }}}\left\{\theta^{\prime}(\mathrm{P})+M_{0}\left|\delta^{\prime}(\mathrm{P})\right|\right\} \leq & c \sum_{\Gamma \in \Gamma} \sum_{\mathrm{P} \in \Gamma} \sigma_{\Gamma}(\mathrm{P}) \leq c\left(\sum_{\Gamma \in \Gamma^{P}}+\sum_{\Gamma \in \Gamma^{S}}\right) \\
& \times\left(\sigma_{\Gamma}\left(\mathrm{P}_{0, \Gamma}\right) \sum_{\mathrm{P} \in \Gamma} c^{k_{\Gamma}(\mathrm{P})-k_{\Gamma}\left(\mathrm{P}_{0, \Gamma}\right)}\right) .
\end{aligned}
$$

In all summations above, we clearly understand that only interactions of type (6)-(10) are involved. Let $m_{\Gamma}$ be the number of points $\mathrm{P} \in \Gamma$; we notice that $m_{\Gamma}$ is finite by Proposition 8.1 .

If $\Gamma$ is a primary path, then $k_{\Gamma}\left(\mathrm{P}_{0, \Gamma}\right)=1, t_{0, \Gamma}=0$; by (7.5), (7.7) we deduce

$$
\begin{aligned}
\sum_{\mathrm{P} \in \Gamma} c^{k_{\Gamma}(\mathrm{P})-k_{\Gamma}\left(\mathrm{P}_{0, \Gamma}\right)} & \leq \sum_{j=1}^{m_{\Gamma}} c^{j-1} \leq \frac{1}{1-c}, \\
\sum_{\Gamma \in \Gamma^{P}} \sigma_{\Gamma}\left(t_{0, \Gamma}\right) & \leq L(0) .
\end{aligned}
$$

If $\Gamma$ is a secondary path, by Lemma 5.3 , we have

$$
\begin{aligned}
\sum_{\mathrm{P} \in \Gamma} c^{k_{\Gamma}(\mathrm{P})-k_{\Gamma}\left(\mathrm{P}_{0, \Gamma}\right)} & \leq \sum_{j=0}^{m_{\Gamma}} c^{j} \leq \frac{1}{1-c}, \\
\sum_{\Gamma \in \Gamma^{S}} \sigma_{\Gamma}\left(t_{0, \Gamma}\right) & \leq 3 \epsilon \sum_{\mathrm{P}} Q(\mathrm{P}) \leq F(O) .
\end{aligned}
$$

The claim (7.13) follows. Since $J^{\prime}$ is an arbitrary space-like curve, we proved the lemma.

By Lemma 4.3, the total amount of rarefaction waves generated by interactions of type (3) is bounded from above by

$$
D \sum_{\text {P: Case (3) }} \alpha_{1}(\mathrm{P}) \alpha_{2}(\mathrm{P})=\sum_{\mathrm{P}: \text { Case (3) }} Q(\mathrm{P}) .
$$

Condition (5.14) implies $M_{0} D_{2} \leq D$; then, also the total amount of entropy paths generated by an interaction of type (3) is estimated by the right-hand side of (7.16). Thus, we have proved the following result. 
Proposition 7.3 The total amount of rarefaction waves and entropy paths generated by an interaction of type (3) is less than the right-hand side of (7.10).

Remark 7.1 The strength of a rarefaction wave may increase only in Cases (2) or (5). Otherwise, either it does not change (Case (11)) or decreases (Cases (7), (9), (10)).

New rarefaction waves are only generated in Cases (3), (4), (5), and (10). We recall that rarefactions generated in Cases (4), (5), and (10) are called secondary rarefactions. We observe that in Case (3), the generated rarefaction wave is only estimated by $Q(\mathrm{P})$ and not by $\epsilon Q(\mathrm{P})$.

We first analyze Case (3); the next proposition provides a result that is slightly stronger than that of Proposition 7.3 and Lemma 6.1 in [3]. Notice that we estimate as well the amount of paths generated in the interaction and associated with entropy waves.

Proposition 7.4 Let $k \geq 2$ and $t$ not an interaction time. The total amount of rarefaction waves of order larger than or equal to $k$, which are generated by an interaction of type (3) in the time interval $[0, t]$, is less than

$$
\frac{D \mathcal{F}(0)}{2(1-c)^{2}}\left\{\frac{c^{k-2}}{1-c} L(0)+V_{k-1}^{S}(t)\right\} .
$$

The total amount of entropy paths whose generation order is larger than or equal to $k$ and are generated by an interaction of type (3) is less than (7.17), with $D_{2}$ replacing $D$.

Proof We denote by $\left\{\mathrm{P}_{m}\right\}$ the collection of all interaction points in the time interval $[0, t]$ where

$$
\text { Case (3) occurs and } \max \left\{k_{\alpha_{1}\left(\mathrm{P}_{m}\right)}, k_{\alpha_{2}\left(\mathrm{P}_{m}\right)}\right\} \geq k-1 \text {. }
$$

Here, $\alpha_{j}\left(\mathrm{P}_{m}\right)$, for $j=1,2$, are the strengths of the shock waves incoming at $\mathrm{P}_{m}$, analogously to the notation above. The rarefaction waves (and the entropy paths) of the statement are precisely those generated at such points $\mathrm{P}_{m}$. By Lemma 4.3 and the definition (6.4), the total amount of these rarefaction waves (resp., entropy paths) is bounded from above by

$$
D \sum_{\mathrm{P}_{m}} \alpha_{1}\left(\mathrm{P}_{m}\right) \alpha_{2}\left(\mathrm{P}_{m}\right)
$$

In the case of entropy paths, simply replace $D$ above with $D_{2}$. Then, we must prove that (7.19) is less than (7.17). The same argument also covers the case of the entropy paths.

Let $\boldsymbol{\Gamma}_{\alpha_{j}}\left(\mathrm{P}_{m}\right)$ denote the collection of the paths composing $\alpha_{j}\left(\mathrm{P}_{m}\right)$, for $j=1,2$; the strength of a path $\Gamma_{j} \in \Gamma_{\alpha_{j}}\left(\mathrm{P}_{m}\right)$ is denoted by $\sigma_{\Gamma_{j}}\left(\mathrm{P}_{m}\right)$. By Lemma 7.1 we have

$$
\begin{aligned}
\sum_{\mathrm{P}_{m}} \alpha_{1}\left(\mathrm{P}_{m}\right) \alpha_{2}\left(\mathrm{P}_{m}\right) & \leq \sum_{\mathrm{P}_{m}} \sum_{\Gamma_{1} \in \Gamma_{\alpha_{1}}\left(\mathrm{P}_{m}\right)} \sum_{\Gamma_{2} \in \Gamma_{\alpha_{2}}\left(\mathrm{P}_{m}\right)} \sigma_{\Gamma_{1}}\left(\mathrm{P}_{m}\right) \sigma_{\Gamma_{2}}\left(\mathrm{P}_{m}\right) \\
& \leq \frac{1}{2} \sum_{\Gamma \in \Gamma} \sum_{\mathrm{P}_{m} \in \Gamma} \sigma_{\Gamma}\left(\mathrm{P}_{m}\right) \sum_{\Gamma^{*} \in \Gamma^{*}\left(\Gamma, \mathrm{P}_{m}\right)} \sigma_{\Gamma^{*}}\left(\mathrm{P}_{m}\right),
\end{aligned}
$$

where $\Gamma^{*}\left(\Gamma, \mathrm{P}_{m}\right)$ is the set of the paths $\Gamma^{*}$ interacting with the path $\Gamma$ at $\mathrm{P}_{m}$. Fix a path $\Gamma$; since clearly $\bigcup_{\mathrm{P}_{m} \in \Gamma} \Gamma^{*}\left(\Gamma, \mathrm{P}_{m}\right) \subset \Gamma \backslash\{\Gamma\}$, by reversing the order of summations we find that

$$
\sum_{\mathrm{P}_{m} \in \Gamma} \sigma_{\Gamma}\left(\mathrm{P}_{m}\right) \sum_{\Gamma^{*} \in \Gamma^{*}\left(\Gamma, \mathrm{P}_{m}\right)} \sigma_{\Gamma^{*}}\left(\mathrm{P}_{m}\right) \leq \sum_{\Gamma^{*} \in \Gamma \backslash\{\Gamma\} \mathrm{P}_{m} \in \Gamma \cap \Gamma^{*}} \sum_{\Gamma^{\prime}}\left(\mathrm{P}_{m}\right) \sigma_{\Gamma^{*}}\left(\mathrm{P}_{m}\right) .
$$


Now, we consider any two points $\mathrm{P}_{m}, \mathrm{P}_{m}^{\prime} \in \Gamma \cap \Gamma^{*}$, with $\mathrm{P}_{m} \neq \mathrm{P}_{m}^{\prime}$, and suppose that $\mathrm{P}_{m}^{\prime}$ is consecutive to $\mathrm{P}_{m}$. By this, we mean there is no point of the collection $\left\{\mathrm{P}_{m}\right\}$ belonging to $\Gamma \cap \Gamma^{*}$ and lying between $\mathrm{P}_{m}$ and $\mathrm{P}_{m}^{\prime}$; moreover, $\mathrm{P}_{m}^{\prime}$ lies after $\mathrm{P}_{m}$. It is impossible that both $k_{\Gamma}\left(\mathrm{P}_{m}\right)=k_{\Gamma}\left(\mathrm{P}_{m}^{\prime}\right)$ and $k_{\Gamma^{*}}\left(\mathrm{P}_{m}\right)=k_{\Gamma^{*}}\left(\mathrm{P}_{m}^{\prime}\right)$ occur. Then, if we move from $\mathrm{P}_{m}$ to $\mathrm{P}_{m}^{\prime}$ along the path $\Gamma$ or $\Gamma^{*}$, either the generation order of $\Gamma$ or $\Gamma^{*}$ increases by at least two or those of both $\Gamma$ and $\Gamma^{*}$ increase by at least one. Hence, by Lemma 7.2, we have

$$
\begin{aligned}
\sum_{\mathrm{P}_{m} \in \Gamma \cap \Gamma^{*}} \sigma_{\Gamma}\left(\mathrm{P}_{m}\right) \sigma_{\Gamma^{*}}\left(\mathrm{P}_{m}\right) & \leq \sum_{j \geq 1} c^{2(j-1)} \sigma_{\Gamma}\left(\mathrm{P}_{0}\right) \sigma_{\Gamma^{*}}\left(\mathrm{P}_{0}\right) \\
& \leq \frac{1}{1-c^{2}} \sigma_{\Gamma}\left(\mathrm{P}_{0}\right) \sigma_{\Gamma^{*}}\left(\mathrm{P}_{0}\right)
\end{aligned}
$$

where, with a slight abuse of notation, we denoted by $\mathrm{P}_{0}$ the first point of the $\mathrm{P}_{m}$ 's in $\Gamma \cap \Gamma^{*}$. By (7.18), we have either $k_{\Gamma}\left(\mathrm{P}_{0}\right) \geq k-1$ or $k_{\Gamma^{*}}\left(\mathrm{P}_{0}\right) \geq k-1$ (of course both possibilities may occur). In order to simplify the notation in the proof, we assume that the first possibility always occurs, namely that $k_{\Gamma}\left(\mathrm{P}_{0}\right) \geq k-1$, otherwise simply replace $\Gamma$ with $\Gamma^{*}$. Under the notation above, we find the estimate

$$
\sum_{\Gamma, \Gamma^{*} \in \boldsymbol{\Gamma}} \sigma_{\Gamma}\left(\mathrm{P}_{0}\right) \sigma_{\Gamma^{*}}\left(\mathrm{P}_{0}\right) \leq \frac{\mathcal{F}(0)}{1-c}\left\{\frac{c^{k-2}}{1-c} L(0)+\mathcal{F}(0)\right\} .
$$

Above, we split the sums into primary and secondary paths associated with shock waves; the total amount of the former is bounded by $L^{-}(0)$ that of the latter by $F(0)$, because of (7.6). Together, they are bounded by $\mathcal{F}(0)$. The second factor is deduced by $(7.8)$ and the definition of $V_{k-1}^{S}(t)$.

This proves that (7.19) is less than (7.17) and concludes the proof.

Remark 7.2 We notice that (7.17) can be bounded as follows, by using (7.6):

$$
\frac{D \mathcal{F}(0)}{2(1-c)^{2}}\left\{\frac{c^{k-2}}{1-c} L(0)+V_{k-1}^{S}(t)\right\} \leq \frac{D \mathcal{F}(0)^{2}}{2(1-c)^{3}} .
$$

A by-product of Proposition 7.4 concerns the total amount of rarefaction waves produced by an interaction of type (3): it suffices to take $k=2$ in (7.17) or simply the right-hand side of (7.23). These bounds must be considered together with that already provided in Proposition 7.3 .

Cases (2) and (5) are considered below. We denote by $\xi(t)$ the continuation of the rarefaction $\xi$ for times $t$ beyond an interaction time $t_{1}$. Therefore, for instance, in Case (5), we have that $\xi(t)=\xi^{\prime}$ just after the interaction and so on.

Proposition 7.5 Suppose that a 1-rarefaction wave $\xi$ interacts at $t=t_{1}$ with either a 2 shock or an entropy wave $\sigma$. Let $\Gamma$ be a path to which the front of $\sigma$ belongs. Then, no front of 2-shock or entropy wave interacting with $\xi(t)$ at $t>t_{1}$ belongs to $\Gamma$.

Proof Let $t_{2}>t_{1}$ be the first time at which $\xi(t)$ interacts with a wave $\sigma^{\prime}$ whose front belongs to $\Gamma$; the wave $\sigma^{\prime}$ necessarily is a 1 -shock wave.

If the 1 -wave that outcomes from the interaction at $t_{2}$ is a shock, then $\xi(t)$ no longer exists for $t>t_{2}$. If it is a rarefaction, then the path $\Gamma$ is continued along the 2 -shock front or entropy front; hence, the path does not $\operatorname{cross} \xi(t)$. 
We denote

$$
\bar{D}=\frac{D_{1}}{M_{0}}\left(1+\frac{D G^{*}}{8\left(1-c^{3}\right)}\right) .
$$

Proposition 7.6 Consider a rarefaction wave $\xi$ at $t=t_{1}$. For $t>t_{1}$, we have

$$
\xi(t) \leq e^{\epsilon \bar{D} \mathcal{F}(0)} \xi
$$

Proof For either a shock or an entropy wave $\sigma$, we introduce the notation

$$
D_{\sigma}= \begin{cases}D & \text { if } \sigma=\alpha, \beta \\ D_{1} & \text { if } \sigma=\delta\end{cases}
$$

Let the 1-rarefaction $\xi^{\prime}$ be the continuation of $\xi$ after the interaction of $\xi$ with either a 2-shock wave (Case (2)) or an entropy wave (Case (5)) $\sigma$, see Remark 7.1. By Lemma 4.3, we find that

$$
\xi^{\prime} \leq\left(1+\epsilon D_{\sigma}|\sigma|\right) \xi
$$

Hence, recalling what we pointed out before Proposition 7.5, we have

$$
\xi(t) \leq \prod_{\sigma=\beta, \delta}\left(1+\epsilon D_{\sigma}|\sigma|\right) \xi \leq e^{\epsilon \sum_{\sigma=\beta, \delta} D_{\sigma}|\sigma|} \xi,
$$

where the product and the sum are performed over all waves $\beta$ or $\delta$ which interact with $\xi\left(t^{\prime}\right)$ at times $t^{\prime}<t$ in an interaction of type (2) or (5), respectively. We observe that every shock or entropy wave in that sum occurs there only once, because of Proposition 7.5.

We now consider the sum in (7.25). First, we observe that $D \leq \frac{D_{1}}{M_{0}}$, because of (5.28). We decompose the shock waves into primary and secondary paths; we do the same for entropy waves but, in this case, we also need to take into account the rarefaction waves generated in Case (3), see Proposition 7.4. We obtain, by (7.23),

$$
\sum_{\sigma=\beta, \delta} D_{\sigma}|\sigma| \leq \frac{D_{1}}{M_{0}}\left\{\mathcal{F}(0)+\frac{D \mathcal{F}(0)^{2}}{2(1-c)^{3}}\right\} \leq \frac{D_{1}}{M_{0}} \mathcal{F}(0)\left\{1+\frac{D G^{*}}{8\left(1-c^{3}\right)}\right\}
$$

and then (7.24) follows by (7.25).

We denote by $\mathcal{R}_{k}(t)\left(\mathcal{W}_{k}(t)\right)$ the total amount of the strengths of all rarefaction waves (of all waves, respectively) having generation order larger than or equal to $k$ at time $t$.

Proposition 7.7 If $k \geq 2$, we have

$$
\begin{aligned}
\mathcal{R}_{k}(t) \leq & \frac{D \mathcal{F}(0) e^{\epsilon \bar{D} \mathcal{F}(0)}}{2(1-c)\left(1-c^{2}\right)}\left\{\frac{c^{k-2}}{1-c} L(0)+V_{k-1}^{S}(t)\right\}+V_{k}^{S}(t), \\
\mathcal{W}_{k}(t) \leq & \frac{c^{k-1}}{1-c} L(0)+V_{k}^{S}(t) \\
& +\frac{D \mathcal{F}(0) e^{\epsilon \bar{D} \mathcal{F}(0)}}{2(1-c)\left(1-c^{2}\right)}\left\{\frac{c^{k-2}}{1-c} L(0)+V_{k-1}^{S}(t)\right\} .
\end{aligned}
$$

Proof We recall that the generation order of a rarefaction wave does not increase in time after an interaction; as a consequence, the rarefaction waves generated at time $0+$ keep their generation order 1. Then, (7.26) follows by Propositions 7.4, 7.6 and the definition of $V_{k}^{S}(t)$.

In order to prove (7.27), we also need to take into account shock and entropy waves. Then, (7.27) follows by (7.6) and (7.26). 


\section{Stability of wave-front tracking scheme}

First, following Bressan [7], we prove that the approximate solution constructed according to the algorithm in Sect. 3 is defined for all $0 \leq t<\infty$.

Proposition 8.1 Let $\mathcal{I}$ be the set of interaction points of an approximate solution. Then, the accurate Riemann solver is used at most a finite number of times in $\mathcal{I}$. As a consequence, the approximate solution is defined for all $0 \leq t<\infty$.

Proof The accurate Riemann solver is used when the strengths of the interacting waves satisfy $\left|\theta^{\prime} \theta^{\prime \prime}\right| \geq \rho$. Lemma 7.4 shows that the number of the interaction points where this occurs is less than $C / \rho$, for a suitable $C$ independent of $\epsilon$. Then, the number of physical fronts is finite. We emphasize that also Lemma 7.4 gives a bound on the number of interaction points, which however depends on $\epsilon$.

Non-physical fronts are possibly generated when two physical fronts interact; moreover, any two physical fronts interact only once. Thus, also the number of non-physical fronts is finite.

Now, we estimate the total amount of secondary paths and secondary rarefaction waves in terms of the quadratic functionals. Let us denote for simplicity

$$
\sum_{\geq k ; \mathcal{A}} \gamma \theta=\sum_{\substack{\max \left\{k_{\gamma}, k_{\theta}\right\} \geq k \\ \gamma, \theta \text { approaching }}} \gamma \theta,
$$

for $k \geq 1$. We define, analogously to (5.4),

$$
\begin{aligned}
Q_{k}(t)= & M_{0} M_{1} \sum_{\geq k ; \mathcal{A}}(\alpha+\beta+\xi+\pi)|\delta|+M_{1} \sum_{\geq k ; \mathcal{A}}(\xi \alpha+\xi \beta+\pi \alpha+\pi \beta) \\
& +M_{2} \sum_{\geq k ; \mathcal{A}}\left(\alpha_{1} \alpha_{2}+\alpha \beta+\beta_{1} \beta_{2}\right),
\end{aligned}
$$

where the summation is over the waves at time $t$. Then, we denote $Q(t)=Q_{1}(t)$, similarly to (5.4). In a complete analogous way, we define $T V_{D}(t)$ and $T V_{H}(t)$ as in (5.6), (5.7). We also define

$$
\mathrm{TV}(t)=T V_{D}(t)+M_{0} T V_{H}(t), \quad \mathrm{Q}_{k}^{ \pm}(t)=\sum_{0<\tau<t}\left[\Delta Q_{k}(\tau)\right]_{ \pm},
$$

where $\Delta Q_{k}(\tau)=Q_{k}(\tau+)-Q_{k}(\tau-)$ and $\tau$ runs over all interaction times; we denoted by $[x]_{ \pm}$the positive and negative parts of $x$. At last, we denote

$$
\begin{gathered}
\widetilde{\mathrm{TV}}=\sup _{t>0} \operatorname{TV}(t-), \quad \widetilde{\mathrm{V}}_{k}^{S}=\sup _{t>0} V_{k}^{S}(t), \quad \widetilde{\mathcal{W}}_{k}=\sup _{t>0} \mathcal{W}_{k}(t), \\
\widetilde{\mathrm{Q}}_{k}^{ \pm}=\sum_{\tau>0}\left[\Delta Q_{k}(\tau)\right]_{ \pm} .
\end{gathered}
$$

We notice that

$$
\mathrm{Q}_{k}^{+}(t)-\mathrm{Q}_{k}^{-}(t)=\sum_{0<\tau \leq t}\left\{\left[\Delta Q_{k}(\tau)\right]_{+}-\left[\Delta Q_{k}(\tau)\right]_{-}\right\}=Q_{k}(t) \geq 0
$$

for $k \geq 2$ and then $\mathrm{Q}_{k}^{-}(t) \leq \mathrm{Q}_{k}^{+}(t)$. The following lemma is a refinement of Lemmas 5.2 and 5.3. 
Lemma 8.1 Assume that a secondary rarefaction wave or a secondary path of strength $\theta$ and order $l \geq 2$ is generated at time $\tau$. Then

$$
\theta \leq \frac{1}{3} \epsilon\left[\Delta Q_{l-1}(\tau)\right]_{-} .
$$

Proof Consider for instance Case (1) and suppose that a secondary path of order $l$ is generated; then either $\alpha$ or $\beta$ has generation order $l-1$. Proceeding as in (5.17) but replacing $\sum$ with $\sum_{\geq l-1 ; \mathcal{A}}$ we find, together with (5.18), that $\Delta Q_{l-1}(\tau) \leq-7 D \alpha \beta$. It follows from Proposition 6.1 that

$$
\theta \leq \epsilon D \alpha \beta \leq-\frac{1}{7} \epsilon \Delta Q_{l-1}(\tau)
$$

The proof in Cases (2)-(5) and (10) is completely analogous.

Proposition 8.2 For $k \geq 2$, we have

$$
V_{k}^{S}(t) \leq e^{\epsilon \bar{D} \mathcal{F}(0)} \frac{\epsilon}{3} \sum_{h=0}^{k-2} c^{h} \mathrm{Q}_{k-h-1}^{-}(t) .
$$

Proof First, suppose that a secondary path $\Gamma^{S}$ of order $l$ is generated at time $\tau<t$. We claim that the contribution of $\Gamma^{S}$ to $V_{k}^{S}(t)$ is less than

$$
\begin{cases}\frac{1}{3} \epsilon c^{h}\left[\Delta Q_{k-h-1}(\tau)\right]_{-} & \text {if } l=k-h \leq k, \\ \frac{1}{3} \epsilon\left[\Delta Q_{k-1}(\tau)\right]_{-} & \text {if } l>k .\end{cases}
$$

If $l \leq k$, then $l=k-h$ for some $0 \leq h \leq k-2$. The path $\Gamma^{S}$ contributes to $V_{k}^{S}(t)$ only if it is continued in the time interval $(\tau, t)$ through at least $h$ interactions. By (7.5) and (8.4), we obtain the first estimate in (8.6).

If $l>k$, the amount of $\Gamma^{S}$ contributing to $V_{k}^{S}(t)$ is less than $\frac{\epsilon}{3}\left[\Delta Q_{l-1}(\tau)\right]_{-} \leq$ $\frac{\epsilon}{3}\left[\Delta Q_{k-1}(\tau)\right]_{-}$again by (8.4). This proves (8.6).

Then, the contribution of the secondary paths to $V_{k}^{S}(t)$ is bounded by

$$
\frac{\epsilon}{3} \sum_{h=0}^{k-2} \sum_{\substack{0<\tau<t: \\ \Gamma^{S} \text { is generated }}} c^{h}\left[\Delta Q_{k-h-1}(\tau)\right]_{-} \leq \frac{\epsilon}{3} \sum_{h=0}^{k-2} c^{h} \mathrm{Q}_{k-h-1}^{-}(t) .
$$

Next, suppose that at time $\tau<t$ a secondary rarefaction wave $\theta$ of order $l$ is generated; it contributes to $V_{k}^{S}(t)$ if and only if $l \geq k$. In this case, we have, by (8.4),

$$
\theta \leq \frac{1}{3} \epsilon\left[\Delta Q_{l-1}(\tau)\right]_{-} \leq \frac{1}{3} \epsilon\left[\Delta Q_{k-1}(\tau)\right]_{-} .
$$

By (7.24) and (8.8), the contribution of the secondary rarefaction waves (2nd RW) to $V_{k}^{S}(t)$ is bounded by

$$
e^{\epsilon \bar{D} \mathcal{F}(0)} \frac{\epsilon}{3} \sum_{\substack{0<\tau<t: \\ \text { 2nd RW is generated }}}\left[\Delta Q_{k-1}(\tau)\right]_{-} \leq e^{\epsilon \bar{D} \mathcal{F}(0)} \frac{\epsilon}{3} \mathrm{Q}_{k-1}^{-}(t) .
$$

Above, the sum is performed over all times $0<\tau<t$ where a secondary rarefaction wave is generated. Thus, (8.5) follows by (8.7) and (8.9). 
Next, we study the variation of $Q_{k}(t)$. For $k \geq 1$, let $I_{k}$ denote the set of times where two waves $\gamma, \theta$ with $\max \left\{k_{\gamma}, k_{\theta}\right\}=k$ interact. The interaction patterns in Lemma 4.3 are denoted by

$W_{1} W_{2}$ : a 1-wave and a 2-wave, Cases (1), (2);

$W_{0} W: \quad$ a 1 or 2-wave and an entropy wave, Cases (4), (5);

$S_{i} S_{i}$ : two shock waves of the same family, Case (3);

$S_{i} R_{i}$ : a shock wave and a rarefaction wave of the same family, Cases (6)-(10).

We notice that Case (11) is trivial. In the following Lemma 8.2, we denote $\theta^{\prime}=\beta^{\prime}$ in Cases (6)-(9) and $\theta^{\prime}=\pi^{\prime}$ in Case (10), as in the previous section. The estimates (8.10)(8.12) are obtained by summing up the estimates obtained in the possible interactions and must be read as follows: referring to (8.10), for example, in an interaction $W_{1} W_{2}$ or $W_{0} W$ $\left(S_{i} S_{i}, S_{i} R_{i}\right)$ only the first (second, third, respectively) summand in braces appears. The notation $S_{i} R_{i}\left(k_{\alpha}<k-1\right)$ in (8.11) and (8.12) refers to the case $S_{i} R_{i}$ when the order of the incoming shock wave is less then $k-1$.

Lemma 8.2 Let $t$ be an interaction time and $\mathrm{P}$ the point where the interaction occurs. We have the following estimates:

(i) if $t \in I_{1} \cup \cdots \cup I_{k-2}$, for $k \geq 3$, then

$$
\Delta Q_{k}(t) \leq M_{3}\left\{3 \epsilon Q(\mathrm{P})+3 D \alpha_{1} \alpha_{2}+\theta^{\prime}+M_{0}\left|\delta^{\prime}\right|\right\} \mathcal{W}_{k}(t-) ;
$$

(ii) if $t \in I_{k-1}$, for $k \geq 2$, then

$$
\begin{aligned}
& \Delta Q_{k}(t) \quad\left\{\begin{array}{c}
M_{3}\left\{\epsilon\left[\Delta Q_{k-1}(t)\right]_{-}+3 D \alpha_{1} \alpha_{2}\right\} \operatorname{TV}(t-) \\
+M_{3}\left(\theta^{\prime}+M_{0}\left|\delta^{\prime}\right|\right) \mathcal{W}_{k}(t-) \quad S_{i} R_{i}\left(k_{\alpha}<k-1\right) \\
M_{3}\left\{\epsilon\left[\Delta Q_{k-1}(t)\right]_{-}+3 D \alpha_{1} \alpha_{2}\right\} \operatorname{TV}(t-) \\
+M_{3}\left(\theta^{\prime}+M_{0}\left|\delta^{\prime}\right|\right) \operatorname{TV}(t-) \quad \text { otherwise } ;
\end{array}\right.
\end{aligned}
$$

(iii) if $t \in I_{k} \cup I_{k+1} \cup \cdots$, for $k \geq 1$, then

$$
\Delta Q_{k}(t) \leq\left\{\begin{array}{cl}
M_{3}\left(\theta^{\prime}+M_{0}\left|\delta^{\prime}\right|\right) \mathcal{W}_{k}(t-) & \\
+\alpha \xi\left(\epsilon M_{3} D \operatorname{TV}(t-)-M_{1}\right) & S_{i} R_{i}\left(k_{\alpha}<k-1\right) \\
M_{3}\left(\theta^{\prime}+M_{0}\left|\delta^{\prime}\right|\right) \operatorname{TV}(t-) & \text { otherwise. }
\end{array}\right.
$$

Proof If $t \in I_{1} \cup \cdots \cup I_{k-2}, k \geq 3$, by Lemma 4.3, (5.14), (7.6) and the notation introduced before (7.27) we have

$$
\Delta Q_{k}(t) \leq \begin{cases}3 \epsilon M_{3} Q(\mathrm{P}) \mathcal{W}_{k}(t-) & : W_{1} W_{2}, W_{0} W \\ 3 D M_{3} \alpha_{1} \alpha_{2} \mathcal{W}_{k}(t-) & : S_{i} S_{i} \\ M_{3}\left(\theta^{\prime}+M_{0}\left|\delta^{\prime}\right|\right) \mathcal{W}_{k}(t-): & S_{i} R_{i}\end{cases}
$$

Hence, the estimate (8.10) follows by summing up the lines above.

If $t \in I_{k-1}, k \geq 2$, by (4.6), we have

$$
\Delta Q_{k}(t) \leq \begin{cases}\epsilon M_{3}\left[\Delta Q_{k-1}(t)\right]-\operatorname{TV}(t-) & : W_{1} W_{2}, W_{0} W \\ 3 D M_{3} \alpha_{1} \alpha_{2} \operatorname{TV}(t-) & : S_{i} S_{i} \\ M_{3}\left(\beta^{\prime}+M_{0}\left|\delta^{\prime}\right|\right) \mathcal{W}_{k}(t-) & \\ \quad+\epsilon M_{3} D \alpha \xi \operatorname{TV}(t-) & : S_{i} R_{i}\left(k_{\alpha}<k-1\right) \\ M_{3}\left(\theta^{\prime}+M_{0}\left|\delta^{\prime}\right|\right) \operatorname{TV}(t-) & : S_{i} R_{i}\left(k_{\alpha}=k-1\right),\end{cases}
$$


whence (8.11). We notice that the additional summand $\epsilon M_{3} D \alpha \xi \mathrm{TV}(t-)$ is only due to Case (10).

Finally, we consider the case $t \in I_{k} \cup I_{k+1} \cup \cdots, k \geq 1$. Recalling the proof of Lemma 5.2 , we deduce that

$$
\Delta Q_{k}(t) \leq \begin{cases}0 & : W_{1} W_{2}, W_{0} W, S_{i} S_{i} \\ \left(M_{3} \theta^{\prime}+M_{1} M_{0}\left|\delta^{\prime}\right|\right) \mathcal{W}_{k}(t-) & \\ +\alpha \xi\left(\epsilon M_{3} D \operatorname{TV}(t-)-M_{1}\right) & : S_{i} R_{i}\left(k_{\alpha}<k-1\right) \\ \left(M_{3} \theta^{\prime}+M_{1} M_{0}\left|\delta^{\prime}\right|\right) \operatorname{TV}(t-) & : S_{i} R_{i}\left(k_{\alpha}=k-1\right)\end{cases}
$$

The proof is complete.

Now, we carry out an iterative estimate for the quadratic functional $\widetilde{\mathrm{Q}}_{k}^{+}$, see (8.2). To this aim, we define

$$
\kappa=\epsilon M_{3} \widetilde{\mathrm{TV}}<1 \quad \text { and } \quad \kappa^{\prime}=\epsilon M_{3} \mathcal{F}(0)<1 .
$$

Proposition 8.3 For $k \geq 2$, we have

$$
\begin{aligned}
\widetilde{\mathrm{Q}}_{k}^{+} \leq \kappa & \widetilde{\mathrm{Q}}_{k-1}^{+}+\left(\frac{1+c}{1-c}+\frac{3 D}{2} \mathcal{F}(0)\right) M_{3} \widetilde{\mathcal{W}}_{k} \mathcal{F}(0) \\
& +\left(c^{k-2} L(0)+\widetilde{\mathrm{V}}_{k-1}^{S}\right)\left(c+\frac{3 D}{2} \mathcal{F}(0)\right) \frac{M_{3}}{1-c} \widetilde{\mathrm{TV}}
\end{aligned}
$$

Proof We may assume that at each interaction time, there is precisely one interaction [7]; then, each interaction time $\tau$ uniquely corresponds to an interaction point $\mathrm{P}_{\tau}$. It follows from Lemma 8.2 that, for $k \geq 3$,

$$
\begin{aligned}
\widetilde{\mathrm{Q}}_{k}^{+} \leq & M_{3} \widetilde{\mathcal{W}}_{k} \sum_{I_{1} \cup \ldots \cup I_{k-2}}\left\{3 \epsilon Q\left(\mathrm{P}_{\tau}\right)+3 D \alpha_{1} \alpha_{2}+\theta^{\prime}+M_{0}\left|\delta^{\prime}\right|\right\} \\
& +M_{3} \widetilde{\mathrm{TV}} \sum_{I_{k-1}}\left\{\epsilon\left[\Delta Q_{k-1}(\tau)\right]_{-}+3 D \alpha_{1} \alpha_{2}\right\} \\
& +M_{3} \widetilde{\mathrm{TV}} \sum_{I_{k-1} \cup I_{k} \cup \ldots}^{* *}\left\{\theta^{\prime}+M_{0}\left|\delta^{\prime}\right|\right\}+M_{3} \widetilde{\mathcal{W}}_{k} \sum_{I_{k-1} \cup I_{k} \cup \ldots}^{*}\left\{\theta^{\prime}+M_{0}\left|\delta^{\prime}\right|\right\} .
\end{aligned}
$$

The summations are made over all interacting times $\tau$, and the estimates must be read as we explained above Lemma 8.2. Moreover, we denoted with $*$ the summation related to the case $S_{i} R_{i}\left(k_{\alpha}<k-1\right)$ and with $* *$ otherwise, see (8.11), (8.12).

Now, we estimate the sums appearing in the above formula. First, by Lemma 5.3, we deduce

$$
3 \epsilon \sum_{I_{1} \cup \ldots \cup I_{k-2}} Q\left(\mathrm{P}_{\tau}\right) \leq F(0) .
$$

On the other hand, by (8.3), we find that

$$
\sum_{I_{k-1}}\left[\Delta Q_{k-1}(\tau)\right]_{-} \leq \sum_{\tau}\left[\Delta Q_{k-1}(\tau)\right]_{-} \leq \sum_{\tau}\left[\Delta Q_{k-1}(\tau)\right]_{+}=\widetilde{\mathrm{Q}}_{k-1}^{+}
$$


Next, we claim that

$$
\begin{aligned}
& \sum_{I_{k-1} \cup I_{k} \cup \ldots}^{* *}\left(\theta^{\prime}+M_{0}\left|\delta^{\prime}\right|\right) \leq \frac{c}{1-c}\left(c^{k-2} L(0)+\widetilde{\mathrm{V}}_{k-1}^{S}\right), \\
& \sum_{I_{1} \cup \cdots \cup I_{k-2}}\left(\theta^{\prime}+M_{0}\left|\delta^{\prime}\right|\right)+\sum_{I_{k-1} \cup I_{k} \cup \ldots}^{*}\left(\theta^{\prime}+M_{0}\left|\delta^{\prime}\right|\right) \leq \frac{c}{1-c} \mathcal{F}(0),
\end{aligned}
$$

and also that

$$
\begin{aligned}
\sum_{I_{1} \cup \ldots \cup I_{k-2}} \alpha_{1} \alpha_{2} & \leq \frac{\mathcal{F}(0)^{2}}{2} \\
\sum_{I_{k-1}} \alpha_{1} \alpha_{2} & \leq \frac{\mathcal{F}(0)}{2}\left(\frac{c^{k-2}}{1-c} L(0)+\widetilde{\mathrm{V}}_{k-1}^{S}\right) .
\end{aligned}
$$

The estimate (8.14) shall follow by the above estimates.

We first consider (8.15). We define $\mathcal{I}_{h}=\left\{\mathrm{P}_{\tau} ; \tau \in I_{h}\right\}$ and proceed almost exactly as in proving (7.14) in Lemma 7.4. We have

$$
\sum_{\mathrm{P} \in \mathcal{I}_{k-1} \cup \mathcal{I}_{k} \cup \ldots}^{* *}\left\{\theta^{\prime}(\mathrm{P})+M_{0}\left|\delta^{\prime}(\mathrm{P})\right|\right\} \leq c \sum_{\Gamma \in \Gamma} \sum_{\mathrm{P} \in \Gamma \cap\left(\mathcal{I}_{k-1} \cup \mathcal{I}_{k} \cup \cdots\right)}^{* *} \sigma_{\Gamma}(\mathrm{P}) .
$$

For every $\mathrm{P} \in \Gamma \cap\left(\mathcal{I}_{k-1} \cup \mathcal{I}_{k} \cup \cdots\right)$, we have $k_{\Gamma}(\mathrm{P}) \geq k-1$ by the definition of strength of a path given above (7.1). Let $m_{\Gamma}$ be the number of points $\mathrm{P} \in \Gamma \cap\left(\mathcal{I}_{k-1} \cup \mathcal{I}_{k} \cup \ldots\right)$. For the collection of primary paths, we have

$$
\begin{aligned}
\sum_{\Gamma \in \Gamma^{P}} \sum_{\mathrm{P} \in \Gamma \cap\left(\mathcal{I}_{k-1} \cup \mathcal{I}_{k} \cup \ldots\right)}^{* *} \sigma_{\Gamma}(\mathrm{P}) & \leq \sum_{\Gamma \in \Gamma^{P}} \sigma_{\Gamma}\left(\mathrm{P}_{0, \Gamma}\right) \sum_{\mathrm{P} \in \Gamma \cap\left(\mathcal{I}_{k-1} \cup \mathcal{I}_{k} \cup \ldots\right)}^{* *} c^{k_{\Gamma}(\mathrm{P})-k_{\Gamma}\left(\mathrm{P}_{0, \Gamma}\right)} \\
& \leq \sum_{\Gamma \in \Gamma^{P}} \sigma_{\Gamma}\left(\mathrm{P}_{0, \Gamma}\right) \sum_{j=k-1}^{m_{\Gamma}} c^{j-1} \leq \frac{L(0) c^{k-2}}{1-c}
\end{aligned}
$$

For secondary paths, we have

$$
\sum_{\Gamma \in \Gamma^{S}} \sum_{\mathrm{P} \in \Gamma \cap\left(\mathcal{I}_{k-1} \cup \mathcal{I}_{k} \cup \ldots\right)} \sigma_{\Gamma}(\mathrm{P}) \leq \widetilde{\mathrm{V}}_{k-1}^{S} .
$$

Then, estimate (8.15) follows.

The proof of (8.16) is analogous: the first sum is estimated as in (7.14), (7.15) and for the second one we exploit (7.8) with $k=2$ and (7.6): the first sum is estimated as in (7.14), (7.15) and for the second one, we exploit (7.8) with $k=2$ and (7.6). More precisely,

$$
\begin{aligned}
& \sum_{I_{1} \cup \cdots \cup I_{k-2}}\left(\theta^{\prime}+M_{0}\left|\delta^{\prime}\right|\right)+\sum_{I_{k-1} \cup I_{k} \cup \ldots}^{*}\left(\theta^{\prime}+M_{0}\left|\delta^{\prime}\right|\right) \\
\leq & \sum_{\mathrm{P} \in \Gamma}\left\{\theta^{\prime}(\mathrm{P})+M_{0}\left|\delta^{\prime}(\mathrm{P})\right|\right\} \\
\leq & c\left(\sum_{\Gamma \in \Gamma^{P}}+\sum_{\Gamma \in \Gamma^{S}}\right)\left(\sigma_{\Gamma}\left(\mathrm{P}_{0, \Gamma}\right) \sum_{\mathrm{P} \in \Gamma} c^{k_{\Gamma}(\mathrm{P})-k_{\Gamma}\left(\mathrm{P}_{0, \Gamma}\right)}\right) .
\end{aligned}
$$


If $\Gamma$ is a primary path, then

$$
\sum_{\mathrm{P} \in \Gamma} c^{k_{\Gamma}(\mathrm{P})-k_{\Gamma}\left(\mathrm{P}_{0, \Gamma}\right)} \leq \sum_{j=1}^{\infty} c^{j-1} \leq \frac{1}{1-c} \quad \text { and } \quad \sum_{\Gamma \in \Gamma^{P}} \sigma_{\Gamma}\left(t_{0, \Gamma}\right) \leq L(0) .
$$

If $\Gamma$ is a secondary path, then

$$
\sum_{\mathrm{P} \in \Gamma} c^{k_{\Gamma}(\mathrm{P})-k_{\Gamma}\left(\mathrm{P}_{0, \Gamma}\right)} \leq \sum_{j=0}^{\infty} c^{j} \leq \frac{1}{1-c} \quad \text { and } \quad \sum_{\Gamma \in \Gamma^{S}} \sigma_{\Gamma}\left(t_{0, \Gamma}\right) \leq \widetilde{\mathrm{V}}_{2}^{S} \leq F(0) .
$$

This proves (8.16).

Next, we prove the estimate (8.18); the proof of (8.17) is analogous. The proof is analogous to that of Proposition 7.4. We have, by Proposition 7.2,

$$
\begin{aligned}
\sum_{P \in \mathcal{I}_{k-1}}\left|\alpha_{1}(\mathrm{P}) \alpha_{2}(\mathrm{P})\right| & \leq \frac{1}{2} \sum_{\Gamma \in \Gamma} \sum_{\mathrm{P} \in \Gamma \cap \mathcal{I}_{k-1}} \sigma_{\Gamma}(\mathrm{P}) \sum_{\Gamma^{\prime} \in \Gamma(\mathrm{P}) \backslash \Gamma} \sigma_{\Gamma^{\prime}}(\mathrm{P}) \\
& \leq \frac{1}{2} \sum_{\Gamma \in \Gamma} \sum_{\Gamma^{\prime} \in \Gamma \backslash \Gamma} \sum_{\mathrm{P} \in \Gamma \cap \Gamma^{\prime} \cap \mathcal{I}_{k-1}} \sigma_{\Gamma}(\mathrm{P}) \sigma_{\Gamma^{\prime}}(\mathrm{P}) \\
& \leq \frac{\mathcal{F}(0)}{2}\left(\sum_{\Gamma \in \Gamma^{P}}+\sum_{\Gamma \in \Gamma^{S}}\right) \sum_{\mathrm{P} \in \Gamma \cap \mathcal{I}_{k-1}} \sigma_{\Gamma}(\mathrm{P}) \\
& \leq \frac{\mathcal{F}(0)}{2}\left(\frac{c^{k-2}}{1-c} L(0)+\widetilde{\mathrm{V}}_{k-1}^{S}\right)
\end{aligned}
$$

by arguing as in proving (8.15). This proves (8.18).

In the following, we assume that $\epsilon \mathrm{TV}(0)$ is sufficiently small, and then $\kappa$ and $\kappa^{\prime}$ in (8.13) are also small. As a consequence, we assume that

$$
e^{\epsilon \bar{D} \mathcal{F}(0)} \leq 2 \text {. }
$$

Proposition 8.4 If $\epsilon \mathrm{TV}(0)$ is sufficiently small, then, for some $c<\lambda_{2}<1$, we have

$$
\widetilde{\mathrm{V}}_{k}^{S}=O(1) \lambda_{2}^{k} .
$$

Proof We denote $\mathrm{Z}_{k}=\epsilon\left(\widetilde{\mathrm{Q}}_{k}^{+}+c \widetilde{\mathrm{Q}}_{k-1}^{+}+\cdots+c^{k-1} \widetilde{\mathrm{Q}}_{1}^{+}\right)$. By (8.21), we deduce

$$
\epsilon \widetilde{\mathrm{Q}}_{k}^{+}=\mathrm{Z}_{k}-c \mathrm{Z}_{k-1}
$$

and by (8.5) and (8.20), we have

$$
\widetilde{\mathrm{V}}_{k}^{S} \leq \frac{2}{3} \mathrm{Z}_{k-1}
$$

Therefore, it is sufficient to prove (8.21) with $\widetilde{\mathrm{V}}_{k}^{S}$ replaced by $\mathrm{Z}_{k-1}$. We plug (8.23) into (7.27) to get

$$
\widetilde{\mathcal{W}}_{k} \leq \frac{c^{k-2}}{1-c} L(0)\left(c+\frac{D \mathcal{F}(0)}{(1-c)\left(1-c^{2}\right)}\right)+\frac{2}{3} \mathrm{Z}_{k-1}+\frac{2 D \mathcal{F}(0)}{3(1-c)\left(1-c^{2}\right)} \mathrm{Z}_{k-2} .
$$

By multiplying (8.14) by $\epsilon$ and taking again into account (8.22), we deduce

$$
\begin{aligned}
\mathrm{Z}_{k}-c \mathrm{Z}_{k-1} \leq & \left(\kappa+\frac{2}{3} B \kappa^{\prime}\right) \mathrm{Z}_{k-1}+\left(-c \kappa+B E \kappa^{\prime}+\frac{2}{3} \frac{\kappa C}{1-c}\right) \mathrm{Z}_{k-2} \\
& +\left(A B \kappa^{\prime}+\frac{L(0) C}{1-c} \kappa\right) c^{k-2},
\end{aligned}
$$


where

$$
\begin{array}{ll}
A=\frac{L(0)}{1-c}\left(c+\frac{D \mathcal{F}(0)}{(1-c)\left(1-c^{2}\right)}\right), & B=\frac{1+c}{1-c}+\frac{3 D \mathcal{F}(0)}{2}, \\
C=c+\frac{3 D \mathcal{F}(0)}{2}, & E=\frac{2 D \mathcal{F}(0)}{3(1-c)\left(1-c^{2}\right)} .
\end{array}
$$

We rewrite inequality (8.24) as

$$
\mathrm{Z}_{k}-a \mathrm{Z}_{k-1}+b \mathrm{Z}_{k-2} \leq d c^{k-2}
$$

for

$$
a=c+\kappa+\frac{2}{3} B \kappa^{\prime}, \quad b=c \kappa-B E \kappa^{\prime}-\frac{2}{3} \frac{\kappa}{1-c} C, \quad d=A B \kappa^{\prime}+\frac{L(0)}{1-c} C \kappa .
$$

If both $\kappa$ and $\kappa^{\prime}$ are sufficiently small, then the equation $\lambda^{2}-a \lambda+b=0$ has two real roots $\left|\lambda_{1}\right|<\lambda_{2}$ with $0<c<\lambda_{2}<1$. Since $\mathrm{Z}_{k} \geq 0$, we deduce from the inequality (8.25)

$$
\mathrm{Z}_{k}=O(1) \lambda_{2}^{k-1}
$$

which proves the proposition.

Proof of Theorem 1.3 The proof is analogous to that of Theorem 2.1 in Asakura-Corli [5]; then, only a sketch is reported here. Estimates of physical waves are obtained by Propositions 7.1 and 8.4. About estimates of non-physical waves, let $\epsilon$ denote an arbitrary nonphysical wave. As in Proposition 11.10 in Asakura-Corli [5], it follows that $|\epsilon| \leq C_{1} \rho$ and

$$
\sum_{\substack{\epsilon \in \mathcal{N} \mathcal{P} \\ k_{\epsilon} \geq k}}|\epsilon| \leq C_{2} \sup _{t \geq 0}\left\{V_{k}^{P}(t)+V_{k}^{S}(t)\right\}
$$

for some positive constants $C_{1}$ and $C_{2}$.

Proposition 8.5 For given $h>0$, there exists $\rho>0$ such that the approximate solution constructed by the front-tracking scheme satisfies

$$
\sum_{\epsilon \in \mathcal{N} \mathcal{P}}|\epsilon| \leq h
$$

Proof The proof goes as in Bressan [7]. Let $N_{0}$ be the number of shock waves at $t=0$. Then, there exists a polynomial $P(\xi, \eta)$ such that, by exploiting the above inequalities, we have

$$
\sum_{\epsilon \in \mathcal{N} \mathcal{P}}|\epsilon|=\sum_{\substack{\epsilon \in \mathcal{N} \mathcal{P} \\ k_{\epsilon} \leq k-1}}|\epsilon|+\sum_{\substack{\epsilon \in \mathcal{N} \mathcal{P} \\ k_{\epsilon} \geq k}}|\epsilon|=O(1) P\left(N_{0}, h^{-1}\right) \rho+O(1) \lambda_{2}^{k}
$$

Hence, we choose $k$ such that $O(1) \lambda_{2}^{k} \leq \frac{h}{2}$ and then $\rho$ so that (8.26) holds.

By Proposition 8.26, we have a uniform bound of non-physical waves and hence of $\operatorname{TV} U^{h}(\cdot, t)$. The existence of a global solution is proved by the usual argument in Bressan [7] and Smoller [20]. 


\section{References}

1. Amadori, D., Corli, A.: On a model of multiphase flow. SIAM J. Math. Anal. 40(1), 134-166 (2008)

2. Asakura, F.: Decay of solutions for the equations of isothermal gas dynamics. Jpn. J. Ind. Appl. Math. 10(1), 133-164 (1993)

3. Asakura, F.: Wave-front tracking for the equations of isentropic gas dynamics. Q. Appl. Math. 63(1), 20-33 (2005)

4. Asakura, F.: Wave-front tracking for the equations of non-isentropic gas dynamics-Proofs of basic lemmas. Acta Math. Vietnam. 38, 487-516 (2013)

5. Asakura, F., Corli, A.: Global existence of solutions by path decomposition for a model of multiphase flow. Q. Appl. Math. 71, 135-182 (2013)

6. Bressan, A.: Global solutions of systems of conservation laws by wave-front tracking. J. Math. Anal. Appl. 170(2), 414-432 (1992)

7. Bressan, A.: Hyperbolic Systems of Conservation Laws. The One-Dimensional Cauchy Problem. Oxford University Press, Oxford (2000)

8. Dafermos, C.M.: Polygonal approximations of solutions of the initial value problem for a conservation law. J. Math. Anal. Appl. 38, 33-41 (1972)

9. Dafermos, C.M.: Hyperbolic Conservation Laws in Continuum Physics, 3rd edn. Springer, Berlin (2010)

10. Fermi, E.: Thermodynamics. Dover, New York (1956)

11. Glimm, J.: Solutions in the large for nonlinear hyperbolic systems of equations. Comm. Pure Appl. Math. 18, 697-715 (1965)

12. Glimm, J., Lax, P.D.: Decay of Solutions of Systems of Nonlinear Hyperbolic Conservation Laws. Memoirs of the American Mathematical Society, No. 101. American Mathematical Society, Providence, RI (1970)

13. Goatin, P., LeFloch, P.G.: $L^{1}$ continuous dependence for the Euler equations of compressible fluids dynamics. Commun. Pure Appl. Anal. 2(1), 107-137 (2003)

14. Holden, H., Risebro, N.H., Sande, H.: The solution of the Cauchy problem with large data for a model of a mixture of gases. J. Hyperbolic Differ. Equ. 6(1), 25-106 (2009)

15. Liu, T.P.: Solutions in the large for the equations of nonisentropic gas dynamics. Indiana Univ. Math. J. 26(1), 147-177 (1977)

16. Nishida, T.: Global solution for an initial boundary value problem of a quasilinear hyperbolic system. Proc. Jpn. Acad. 44, 642-646 (1968)

17. Nishida, T.: Nonlinear Hyperbolic Equations and Related Topics in Fluid Dynamics. Département de Mathématique, Université de Paris-Sud, Orsay, 1978. Publications Mathématiques d'Orsay, No. 78-02

18. Nishida, T., Smoller, J.A.: Solutions in the large for some nonlinear hyperbolic conservation laws. Comm. Pure Appl. Math. 26, 183-200 (1973)

19. Risebro, N.H.: A front-tracking alternative to the random choice method. Proc. Am. Math. Soc. 117(4), 1125-1139 (1993)

20. Smoller, J.: Shock Waves and Reaction-Diffusion Equations, 2nd edn. Springer, New York (1994)

21. Temple, B., Young, R.: The large time stability of sound waves. Comm. Math. Phys. 179(2), 417-466 (1996)

22. Temple, J.B.: Solutions in the large for the nonlinear hyperbolic conservation laws of gas dynamics. J. Differ. Equ. 41(1), 96-161 (1981) 